\title{
BIAS IN FOOD STAMPS PARTICIPATION ESTIMATES IN THE PRESENCE OF MISREPORTING ERROR
}

\author{
by \\ Cathleen $\mathrm{Li}^{\mathrm{i}}$ \\ Department of Statistics \\ The University of Chicago
}

CES 13-13 March, 2013

The research program of the Center for Economic Studies (CES) produces a wide range of economic analyses to improve the statistical programs of the U.S. Census Bureau. Many of these analyses take the form of CES research papers. The papers have not undergone the review accorded Census Bureau publications and no endorsement should be inferred. Any opinions and conclusions expressed herein are those of the author(s) and do not necessarily represent the views of the U.S. Census Bureau. All results have been reviewed to ensure that no confidential information is disclosed. Republication in whole or part must be cleared with the authors.

To obtain information about the series, see www.census.gov/ces or contact Fariha Kamal, Editor, Discussion Papers, U.S. Census Bureau, Center for Economic Studies 2K132B, 4600 Silver Hill Road, Washington, DC 20233, CES.Papers.List@census.gov. 


\begin{abstract}
This paper focuses on how survey misreporting of food stamp receipt can bias demographic estimation of program participation. Food stamps is a federally funded program which subsidizes the nutrition of low-income households. In order to improve the reach of this program, studies on how program participation varies by demographic groups have been conducted using census data. Census data are subject to a lot of misreporting error, both underreporting and over-reporting, which can bias the estimates. The impact of misreporting error on estimate bias is examined by calculating food stamp participation rates, misreporting rates, and bias for select household characteristics (covariates).
\end{abstract}

\footnotetext{
${ }^{\mathrm{i}}$ Any opinions and conclusions expressed herein are those of the author(s) and do not necessarily represent the views of the U.S. Census Bureau. All results have been reviewed to ensure that no confidential information is disclosed.
} 


\section{Introduction}

This paper focuses on how survey misreporting of food stamp receipt can bias demographic estimation of program participation. Food stamps is a federally funded program which subsidizes food and grocery purchases of low-income households. In order to improve the reach of this program, studies on how program participation varies by demographic groups have been conducted using census data. Census data are subject to a lot of misreporting error, both under-reporting and over-reporting, which can bias the estimates. The impact of misreporting error on estimate bias is examined by calculating food stamp participation rates, misreporting rates, and bias for select household characteristics (covariates).

This paper finds that the biases in estimates are limited due to certain characteristics of the misreporting errors. The misreporting errors are often correlated with the covariates, so that the impact of the error on biases does not follow the rules of classic error. Given correlated misreporting errors, the estimate biases can be limited if all or some of these conditions are fulfilled:

1. there is low participation within each demographic group and low over-reporting,

2. the demographic group more likely to be on food stamps is more likely to over-report and,

3. the demographic group less likely to be on food stamps is more likely to under-report.

We will explore the data using univariate linear models for each selected covariate as it will allow us to break down the components of misreporting errors to see whether these conditions are fulfilled. The results will help us understand how the errors bias estimates. Finally, we will generalize the behavior of misreporting errors from the univariate model to a multivariate model, with all the covariates included.

\section{Review of Previous Literature}

Households qualify for the food stamps program if their income and asset levels fall below a certain cutoff. The program targets some of the most vulnerable demographics in our society, with children making up nearly half the recipients ${ }^{1}$. Previous studies showed food stamps have a positive social impact, diminishing the overall poverty gap and the growth in wealth disparities in our society ${ }^{2}$. And the program could be even more effective in combating poverty if it reached more low-income households with children ${ }^{3}$.

Out of the millions of people eligible for the program, around $70 \%$ actually participate in food stamps. ${ }^{4}$ In order to increase the program outreach, studies such as Cunnyham et al. 2008 and Wolkwitz 2008 have been carried out to understand how participation rates vary based on household demographics and characteristics. They found that households with children and households below

\footnotetext{
${ }^{1}$ Characteristics of Supplemental Nutrition Assistance Program (SNAP) Households: Fiscal Year 2010

${ }^{2}$ Bishop J.A. Formby, JP and L. Zeager 1996

${ }^{3}$ Jolliffe, et. al. 2005

${ }^{4}$ Trends in Supplemental Nutrition Assistance Program Participation Rates: FY 2002 to FY 2009
} 
the poverty line are significantly more likely to participate than those without children and those slightly above the poverty line. Other papers such as Meyer and Goerge 2011 found characteristics such as marital status of parents, race of the household, and participation in other subsidy programs to be important factors in determining participation.

The study of food stamp participation across different demographic groups often uses survey responses to determine food stamps receipt by individuals. Demographic survey data, such as the American Community Service (ACS), Current Population Survey (CPS) and Survey of Income and Program Participation (SIPP) are preferred to administrative data because of easier access and more detailed covariates for researchers. However, survey data can be susceptible to error due to misreporting, an error which can cause biases in participation estimates.

A few studies of survey misreporting of food stamp receipt indicate that misreporting is largely due to under-reporting rather than over-reporting. We define under-reporting (false negative) as a household that fails to report food stamp participation given recorded receipt and over-reporting (false positive) as a household that reports food stamp participation given no recorded receipt. According to a review of a number of studies on food stamps misreporting in Bound Brown and Mathiowetz 2001, under-reporting (false negative) is between 12 to $30 \%$ and over-reporting (false positive) is between $0.3 \%$ to $15 \%$. A detailed table summarizing the review is shown in Appendix 1. This review included some notable studies on food stamps misreporting such as Moore, Marquis and Bogen 1996, which described under-reporting of 12-17\% and over-reporting between 2-3\%, and Bollinger and David 1997 which estimated under-reporting around $12 \%$ and over-reporting below $1 \%$. Generally, older surveys have lower error rates, indicating the quality of reporting has gotten worse over time.

Bound et al. offers a few rationales for the misreporting of participation in transfer programs like food stamps. Each of the possible causes of food stamp misreporting errors is listed below, with the associated impact on reporting:

1. Retrieval and recollection errors, especially when households are not consistently on food stamps. With this error, under and over-reporting are equally likely.

2. Confusion due to participation in multiple programs. With this type of misreporting, over and under-reporting are equally likely.

3. Social undesirability of programs. This can lead to under-reporting.

4. Non-reporting, when individuals leave a question or item blank. This can contribute to under-reporting. ${ }^{5}$

The above causes for transfer program misreporting provide an explanation for the greater tendency to under-report in surveys. Over-reporting is caused by retrieval error and confusion with other programs, but under-reporting is equally probable with those causes. Under-reporting also results from other common causes of error, including social desirability and omission.

According to a 2009 paper by Meyer, Mok and Sullivan, the rate of under-reporting can be substantial. The study finds under-reporting to be much higher than over-reporting, such that net

\footnotetext{
${ }^{5}$ Non-reporting can also contribute to over-reporting if blanks are imputed, depending on household characteristic and imputation methods.
} 
under-reporting rates can be used as a good approximation of overall under-reporting for recipients. According to the study, not only have net reporting ${ }^{6}$ rates been low, but they have been declining over time. Both the data from the CPS and CE Survey show a steady decline in reporting rates from the late 1970s to 2000s. The SIPP data also show a decline up to the 1990s but a slight recovery in recent years. Overall, the level of misreporting for food stamps, especially under-reporting, is quite large and appears to be growing over time.

Both the under-reporting and over-reporting of food stamp receipt in survey data can affect the studies of food stamp participation. Typically, these studies would model food stamp receipt on the demographic of the household to determine how characteristics affect the likelihood of receipt. The misreporting of the survey data on receipt could lead to a bias in the determinants of receipt. Bollinger's paper found that the misreporting error can greatly affect the likelihood estimates of food stamp receipt. Using a likelihood function for reported food stamp receipts, he concluded that when it was conditioned on the probability of response error, it produced significantly different results from the naive assumptions of no error. Similarly, in a study using comparable samples for survey and administrative data, Meyer and Goerge rejected the null hypothesis of equality between results. Despite the differences between the administrative and survey models, the authors found that most coefficients significant for the administrative model do not change signs and remain significant in the survey model. This finding suggests that biased estimates may still be reasonable approximations of true coefficients despite survey misreporting.

The precise impact of misreporting on estimate bias is still unclear. Currently, there is a dearth of literature that directly explores the relationship between misreporting error and bias in survey data for food stamps. The conclusion of the paper by Meyer and Goerge suggests that, despite biases, the significance and the signs of estimates tend to be preserved. According to Bound et al, the bias tends to be lower with low participation, as long as under-reporting is greater than over-reporting. The same study states that if both under-reporting and over-reporting is high, this can lead to significant bias in the regression. This paper will further explore these conclusions for bias in the estimates of food stamp participation due to over-reporting and under-reporting.

Furthermore, we will explore whether the misreporting errors are correlated with the key covariates predicting food stamp receipt. According to Hausman, Abrevaya and Scott-Morton, in discreteresponse setting, where misreporting errors are not correlated with the covariates and over-reporting is close to 0 , the coefficients always shrink by a factor proportional to the over-reporting and under-reporting rates ${ }^{7}$. Where the errors are correlated with the covariates, we will explore a few conditions that can limit the size of the bias.

\section{How does measurement error bias estimates (in the linear case)?}

The focus of this discussion is on how misreporting error in surveys can bias models used to estimate the demographic of food stamp participation. The response variable for the model of interest is food

\footnotetext{
${ }^{6}$ the weighted ratio of reported beneficiaries to administrative totals

${ }^{7}$ The shrinkage factor $\mathrm{f}=\left(1-\nu_{01}-\nu_{10}\right)$ where $\nu_{01}$ is the under-reporting rate and $\nu_{10}$ is the over-reporting rate. From this formula we can see that the coefficient sign could change if the sum of under-reporting and over-reporting rates is greater than 1 , but this case is rare.
} 
stamp receipt and the covariates include various characteristics of the household such as poverty level (below and above poverty line), household type (single parent), race, and the age of the head of the household.

We would like to explore how the misreporting error in the response variable can create bias when we estimate participation by the demographic groups listed above. For ease of interpretation, we first explore the theoretical impact of misreporting error on estimate bias in a univariate linear model with food stamp receipt on the left hand side and one of the demographic covariate on the right hand side. We explore this effect under two different assumptions first, when the misreporting error is uncorrelated with the covariate (right hand side variable) and second, when the error is correlated.

\subsection{Theoretical Bias 1: Uncorrelated Misreporting Error}

According to Hausman 2001, in the classic case of errors for continuous response variables, where the misreporting error of the left hand side variable is not correlated with the covariate, the misreporting error would not bias the coefficient estimate, and only increase the variance of the estimate. While with a binary response variables ${ }^{8}$, if the misreporting error is not correlated with the covariate, the errors will shrink the coefficient.

We explain this effect below for a simple univariate model with binary response variables. In this example, the misreporting error in the response variable is not correlated with the covariate, but can be correlated with the true response. Given y is the true binary response variable and y' is the reported binary variable for food stamp receipt,

$$
\begin{gathered}
y_{i}= \begin{cases}1 & \text { if on food stamps } \\
0 & \text { otherwise }\end{cases} \\
y_{i}^{\prime}= \begin{cases}1 & \text { if reported on food stamps } \\
0 & \text { otherwise }\end{cases} \\
\pi=P(y=1) \\
\begin{array}{r}
\nu_{01}=P\left(y^{\prime}=0 \mid y=1\right) \\
\nu_{10}=P\left(y^{\prime}=1 \mid y=0\right) \quad \begin{array}{r}
\text { is overall probability of food stamp receipt } \\
\text { is the probability of under-reporting given receipt }
\end{array}
\end{array}
\end{gathered}
$$

The data is fitted to a binary covariate $\mathrm{x}$ where

$$
x_{i}= \begin{cases}1 & \text { if in demographic group } \\ 0 & \text { otherwise }\end{cases}
$$

$$
\begin{array}{ll}
\pi_{1}=P(y=1 \mid x=1) & \text { is the probability of true food stamp receipt given } x=1 \\
\pi_{0}=P(y=1 \mid x=0) & \text { is the probability of true food stamp receipt given } x=0
\end{array}
$$

\footnotetext{
${ }^{8}$ Hausman et al. 1998
} 
The linear regression of true food stamps receipt $y$ on the covariate $x$ is the following

$$
\begin{aligned}
& y_{i}=\alpha+x_{i} \beta+\epsilon_{i} \\
& \quad \text { where } \\
& \begin{aligned}
& \alpha=\%(x=0) \text { on food stamps } \\
& \beta=\%(x=1)-\%(x=0) \text { on food stamps } \\
& \epsilon_{i}=\text { independent error for } \mathrm{i}
\end{aligned}
\end{aligned}
$$

And the true coefficient calculates the difference between the probability that a household is on food stamps given a household is in demographic group and the probability given otherwise.

$$
\beta=\pi_{1}-\pi_{0}
$$

However, the true value of food stamp is often not observed, instead we have a reported receipt of food stamps $y_{i}^{\prime}$ which indicates whether or not a household reported food stamp receipt in a survey. For the reported data, the simple linear regression of reported food stamp receipt on the covariate is

$$
\begin{aligned}
& y_{i}^{\prime}=\alpha^{*}+x_{i} \beta^{*}+\eta_{i} \\
& \quad \text { where } \\
& \alpha^{*}=\%(x=0) \text { reported on food stamps } \\
& \beta^{*}=\%(x=1)-\%(x=0) \text { reported on food stamps } \\
& \eta_{i}=\text { independent error for } \mathrm{i}
\end{aligned}
$$

And the estimate with misreporting error $\beta^{*}$ can be expressed as the difference between those who reported to be on food stamps given $(\mathrm{x}=1)$ and those who reported on food stamps given $(\mathrm{x}=0)$.

$$
\begin{aligned}
\beta^{*} & =\left(\pi_{1}\left(1-\nu_{01}\right)+\left(1-\pi_{1}\right) \nu_{10}\right)-\left(\pi_{0}\left(1-\nu_{01}\right)+\left(1-\pi_{0}\right) \nu_{10}\right) \\
& =\pi_{1}\left(1-\nu_{01}\right)-\pi_{1} \nu_{10}-\pi_{0}\left(1-\nu_{01}\right)+\pi_{0} \nu_{10} \\
& =\left(\pi_{1}-\pi_{0}\right)\left(1-\nu_{01}-\nu_{10}\right)
\end{aligned}
$$

Even though the misreporting error is not correlated with the covariate, the estimated coefficient $\beta^{*}$ can be biased by misreporting errors. The estimate would shrink by a factor of $\left(1-\nu_{01}-\nu_{10}\right)$ but would preserve the same sign, assuming that the sum of false negative and false positive is less than 1.

\subsection{Theoretical Bias 2: Correlated Misreporting Error}

When the misreporting error is correlated with the covariate, the bias behaves in a less predictable way. Since the probability of misreporting is correlated with both the response variable and the 
covariate, we have to replace $\nu_{01}$ and $\nu_{10}$ with 4 parameters to describe the misreporting error.

$$
\begin{array}{lr}
r_{11}=P\left(y^{\prime}=0 \mid y=1 \& x=1\right) & \text { probability of under-reporting given receipt and } \mathrm{x}=1 \\
r_{10}=P\left(y^{\prime}=0 \mid y=1 \& x=0\right) & \text { probability of under-reporting given receipt and } \mathrm{x}=0 \\
r_{01}=P\left(y^{\prime}=1 \mid y=0 \& x=1\right) & \text { probability of over-reporting given no receipt and } \mathrm{x}=1 \\
r_{00}=P\left(y^{\prime}=1 \mid y=0 \& x=0\right) & \text { probability of over-reporting given no receipt and } \mathrm{x}=0
\end{array}
$$

If the misreporting errors are not correlated with $\mathrm{x}$, then these parameters would just be reduced to $\nu_{01}$ and $\nu_{10}$ since $r_{11}=r_{10}=\nu_{01}$ and $r_{01}=r_{00}=\nu_{10}$.

Using the new parameters which describe correlated misreporting errors, the estimate $\beta^{*}$ is calculated as:

$$
\beta^{*}=\left(\pi_{1}\left(1-r_{11}\right)+\left(1-\pi_{1}\right) r_{01}\right)-\left(\pi_{0}\left(1-r_{10}\right)+\left(1-\pi_{0}\right) r_{00}\right)
$$

The true estimate $\beta$ is still the same, but can be expressed in terms of the new parameters.

$$
\beta=\pi_{1}-\pi_{0}=\left(\pi_{1}\left(1-r_{11}\right)+\pi_{1} r_{11}\right)-\left(\pi_{0}\left(1-r_{10}\right)+\pi_{0} r_{10}\right)
$$

The bias or the difference between the estimated and the true coefficient $\beta^{*}-\beta$ is equal to:

$$
\begin{aligned}
\beta^{*}-\beta= & \left(\pi_{1}\left(1-r_{11}\right)+\left(1-\pi_{1}\right) r_{01}-\pi_{0}\left(1-r_{10}\right)-\left(1-\pi_{0}\right) r_{00}\right) \\
& -\left(\pi_{1}\left(1-r_{11}\right)+\pi_{1} r_{11}-\pi_{0}\left(1-r_{10}\right)-\pi_{0} r_{10}\right) \\
= & \left(\left(1-\pi_{1}\right) r_{01}-\pi_{1} r_{11}\right)-\left(\left(1-\pi_{0}\right) r_{00}-\pi_{0} r_{10}\right)
\end{aligned}
$$

This bias is essentially expressed as the differences of differences. The difference between the total false positive and false negative probability of demographic group $x=0$ subtracted from the difference between the total false positive and false negative probability of demographic group $x=1$.

When misreporting errors are uncorrelated with the covariate, the bias creates a predictable shrinkage proportional to $\left(1-\nu_{01}-\nu_{10}\right)$. That is, the greater the sum of false negative and false positive probability, the greater the bias. Furthermore, when the true estimate is close to 0, then the bias would also be close to 0 . While, with correlated misreporting error, bias can still exist even if the true estimate $\left(\pi_{1}-\pi_{0}\right)$ is close to 0 because over-reporting or under-reporting does not distort estimates of $\pi_{1}$ and $\pi_{0}$ uniformly.

If the true coefficient $\left(\pi_{1}-\pi_{0}\right)$ is significantly different from 0 , when the misreporting error is uncorrelated with the covariate, the bias would increase proportionally to the true estimate. While, with correlated misreporting errors, even if the true coefficient is significantly different from 0 , it is still possible for the bias to be small or close to 0 . This is due to a few cases where the misreporting errors can cancel each other out. Below we explore these cases when bias can be small or absent in the presence of a significant true coefficient and correlated misreporting error. These cases apply only to univariate linear regressions. 
Before, proceeding to these cases, we have to establish the distinction between the terms total probability of false positives/false negative and the conditional probability of underreporting/over-reporting given receipt status (under-reporting/over-reporting rates). The under-reporting/over-reporting rates refer to the probability of misreporting given individual is in demographic group $x$ and has a certain food stamp receipt status. So for the demographic group $x=1$, the over-reporting and under-reporting rates are $r_{01}$ and $r_{11}$, respectively. The total probability of false negative/false positive refers to the probability of under-reporting/over-reporting for all those in the demographic group. So for the demographic group $x=1$, the total probabilities are $\pi_{1} r_{11}$ and $\left(1-\pi_{1}\right) r_{01}$ for false negatives and false positives, respectively.

Minimized Bias Case 1: Bias would be minimized if the total probability of false positive/ false negative in demographic group $x=1$ is equal to the total probability of false positive/false negative in demographic group $x=0$. This would be true under the following sub-conditions:

(1.1) The total probability of false positive (over-reporting) given $\mathrm{x}=1$ is equal to the total probability of false positive given $x=0$.

$$
\left(1-\pi_{0}\right) r_{00} \approx\left(1-\pi_{1}\right) r_{01}
$$

(1.2) The total probability of false negative (under-reporting) given $\mathrm{x}=1$ is equal to the total probability of false negative given the $x=0$

$$
\pi_{0} r_{10} \approx \pi_{1} r_{11}
$$

If probability of being on food stamps given $x=1$ is greater than the probability of being on food stamps given $x=0$,

$$
\pi_{0}<\pi_{1} \Rightarrow 1-\pi_{0}>1-\pi_{1}
$$

then for the sub-conditions to be satisfied, the over-reporting rate of $x=1$ has to be greater than the over-reporting rate of $x=0$

$$
r_{01}>r_{00}
$$

Similarly, the under-reporting rate of $x=1$ has to be less than the under-reporting rate of $x=0$

$$
r_{11}<r_{10}
$$

If the reverse is true $\pi_{0}>\pi_{1}$, then the sub-conditions would be satisfied with $r_{01}<r_{00}$ and $r_{11}>r_{10}$.

Criterion 1: Sub-conditions (1.1) and (1.2) are met only if the demographic group that is more likely to adopt food stamps is also more likely to over-report and less likely to under-report.

If the probability of being on food stamps given $x=1$ is equal to the probability of being on food stamps given $x=0$ or $\pi_{1}=\pi_{0}$, then in order for the sub-conditions (1.1) and (1.2) to be fulfilled, the misreporting error has to be uncorrelated with the covariate. In other words, the probability of under-reporting given $x=1$ has to be equal to the probability of under-reporting given $x=0$

$$
r_{11}=r_{10}=\nu_{01}
$$


Similarly, the over-reporting rate given $x=1$ has to be equal to the over-reporting rate given $x=0$

$$
r_{01}=r_{00}=\nu_{10}
$$

If both equalities are satisfied then the calculated bias is reduced to $\left(\pi_{1}-\pi_{0}\right)\left(1-\nu_{01}-\nu_{10}\right)$, which is equal to 0 since $\pi_{1}=\pi_{0}$.

Minimized Bias Case 2: Bias would be minimized if the total probability of false positive/ false negative in demographic group $x=1$ is equal to the total probability of false positive/false negative in demographic group $x=0$. This would be true under the following sub-conditions:

(2.1) The total probability of false positive and false negative reporting are equal given $x=1$.

$$
\left(1-\pi_{1}\right) r_{01} \approx \pi_{1} r_{11}
$$

(2.2) The total probability of false positive and false negative reporting are equal given $x=0$

$$
\left(1-\pi_{0}\right) r_{00} \approx \pi_{0} r_{10}
$$

If the food stamp participation rate given $\mathrm{x}$ is less than $50 \%$, for the sub-condition to be met, the over-reporting rate given $\mathrm{x}$ has to be greater than the under-reporting rate given $\mathrm{x}$. In other words, if

$$
\pi_{1}<50 \%
$$

then bias would be minimized only if

$$
r_{01}<r_{11}
$$

If food stamp participation is high, above $50 \%$, given $x=1$ or $x=0$, the reverse has to true.

Criterion 2: Sub-conditions (2.1) and (2.2) are met only when over-reporting is less likely than under-reporting if participation rate is low (below 50\%) and the reverse if participation rate is above $50 \%$.

If the probability of being on food stamps given $x=1$ is equal to the probability of being on food stamps given $x=0$ or $\pi_{1}=\pi_{0}$, then in order the sub-conditions (1.1) and (1.2) to be fulfilled, under and over-reporting probability given $\mathrm{x}$ has to be equal for the sub-condition to be satisfied. In other words, if $\pi_{1}=50 \%$, then bias would be minimized if $r_{01}=r_{11}$. If $\pi_{0}=50 \%$, then bias would be minimized if $r_{00}=r_{10}$. If $\pi_{1}=\pi_{1}=50 \%$, then similar to Case 1 , bias would be minimized if misreporting errors are uncorrelated with the covariate.

Criteria 1 and 2 establish pre-conditions necessary for bias to be minimized when misreporting errors correlated with covariates exist. We cannot assume that bias will be minimized just because these criteria are satisfied but they do give us some indication of how the bias could behave in the presence of misreporting errors. 


\section{Data}

Using administrative data and survey data, we will examine how misreporting in the food stamp data affects participation estimates for demographic groups and how the data corresponds to the theoretical framework given above. We select a number of demographic groups for our covariates including poverty level (below and above poverty line), household type (single parent and otherwise), race (white and non-white), and the household age (head of household below and greater or equal to age 50). The covariates included in the analysis were selected based on previous literature in the field. From the conclusions of Bollinger 1997, Meyer and Goerge 2011, and Wolkwitz 2008, we believe that both poverty level and household type (single parent household, households without children) are highly significant determinants of food stamps receipt, and highly correlated with misreporting errors. The Meyer and Goerge paper also suggests that race is a significant determinant of food stamp receipt based on administrative data, but finds the significance of the coefficients inconclusive when using the survey data. Due to the apparent bias of the survey data, we include the variable in our analysis. Finally, Meyer and Goerge discuss the controversy of elderly households as a variable. They cite widely varying conclusions in previous literature on the significance of this variable as a determinant of food stamp receipt. While the significance is inconclusive, their paper suggests the variable is highly correlated with misreporting error. Therefore, this variable would be interesting to include in our analysis.

The individual-level administrative and survey data on food stamps comes from a number of sources. The survey data comes from two separate panels of SIPP (Survey of Income and Program Participation). The first panel is the 2001 SIPP with 9 different waves occurring between 2001-03. The second panel is the 2004 SIPP with 4 different waves between 2004-2005. For each panel, the same households are interviewed and followed up with each wave. For the data analysis, all those households without a household PIK ID, a unique ID given to each individual in the survey, are dropped and each observation is weighted by the inverse probability of having a PIK. The survey data provides information on reported food stamp receipt, and the demographic of the individual household.

The administrative data on true food stamp receipt for Illinois and Maryland comes from two different sources. The Maryland data is from the Client Automated Resource and Eligibility System (CARES), covering 1998 and 2003. The Illinois data comes from Illinois Department of Human Services (DHS), covering 1998 to 2004. Both of these data sets were provided to the Census Bureau.

The sample for our analysis consists of the observations cross-listed between both data-sets. From the survey data, we created binary variables for each covariate of interest, dividing each covariate into two different demographic groups.

- Income: $1=$ below poverty line and $0=$ below poverty line

- Household Type: $1=$ Single Parent Household and 0=Otherwise

- Age of Household Head: $1=$ Above or equal to 50 and $0=$ Below 50

- Race: $1=$ White household $0=$ Nonwhite household

The parameters are calculated off 14,840 observations, with adjusted weights for likelihood of being PIKed totaling 83,630,775. The table below shows the mean and standard deviation of the food 
stamp receipt and reported variables, along with the covariates. The unadjusted (sample) values are calculated off the raw counts from the observation and the adjusted (population) value weights each of the counts by inverse likelihood of receiving a PIK.

\begin{tabular}{|l|c|c|c|c|}
\hline & \multicolumn{2}{|c|}{ variable mean } & \multicolumn{2}{c|}{ std dev } \\
\hline & $\begin{array}{c}\text { Sample } \\
\text { (Unadj) }\end{array}$ & $\begin{array}{c}\text { Population } \\
\text { (Adj) }\end{array}$ & $\begin{array}{c}\text { Sample } \\
\text { (Unadj) }\end{array}$ & $\begin{array}{c}\text { Population } \\
\text { (Adj) }\end{array}$ \\
\hline Actual Food Stamps Receipt & 0.053 & 0.042 & 0.225 & 0.0016 \\
Reported Food Stamps Receipt & 0.067 & 0.055 & 0.251 & 0.0018 \\
Income below poverty line & 0.111 & 0.104 & 0.314 & 0.0026 \\
Single Parent Household & 0.114 & 0.100 & 0.318 & 0.0025 \\
White Household & 0.790 & 0.812 & 0.407 & 0.0033 \\
Elderly Household $(\geq$ Age 50) & 0.492 & 0.507 & 0.500 & 0.0043 \\
\hline
\end{tabular}

The data indicates that $4.2 \%$ of the sampled population actually receives food stamps and $5.5 \%$ are reported to be on food stamps. So, overall, there is a net false positive for reported food stamps participation. The misreporting rates are slightly unusual, compared to the rates given by Meyer and Goerge for ACS and CPS, with under-reporting rates ranging between 31\%-53\% and over-reporting rates between $0.4 \%-1.0 \%$. The table below, with tabulation of weighted counts by those reported in the survey and the actual counts in the administrative data, show underreporting rates given food stamp receipt $\nu_{01}=22.8 \%$ and over-reporting rates given no food stamp receipt $\nu_{10}=22.3 \%$ (in bold). The SIPP data has slightly lower under-reporting rate and higher over-reporting rate.

\begin{tabular}{l|cc|c} 
& \multicolumn{2}{|c|}{ SIPP Report } & \\
Administrative Receipt & No Food Stamps & Food Stamps & Total \\
\hline No Food Stamps & $78,242,608$ & $1,865,577$ & $80,108,185$ \\
& $93.6 \%$ & $2.2 \%$ & $95.8 \%$ \\
& $97.7 \%$ & $2.3 \%$ & $100.0 \%$ \\
& $99.0 \%$ & $40.7 \%$ & $95.8 \%$ \\
& 13675 & 372 & 14047 \\
\hline Food Stamps & 803,748 & $2,718,84$ & $3,522,590$ \\
& $1.0 \%$ & $3.3 \%$ & $4.2 \%$ \\
& $22.8 \%$ & $77.2 \%$ & $100.0 \%$ \\
& $1.0 \%$ & $59.3 \%$ & $4.2 \%$ \\
& 165 & 628 & 793 \\
\hline Total & $79,046,357$ & $4,584,419$ & $83,630,775$ \\
& $94.5 \%$ & $5.5 \%$ & $100.0 \%$ \\
& $94.5 \%$ & $5.5 \%$ & $100.0 \%$ \\
& $100.0 \%$ & $100.0 \%$ & $100.0 \%$ \\
& 13840 & 1000 & 14840
\end{tabular}

Table 1: The entries in each cell from top to bottom are population estimate, overall \%, row \%, column $\%$ and sample counts. Population estimates are weighted by household weight adjusted for PIK probability. 


\section{Analysis}

Comparing the results between the administrative data and survey response, we can evaluate the size of misreporting error and determine whether the misreporting errors are correlated with the covariates (demographic groups) of interest. The latter will be measured with a $\chi^{2}$ testing the null hypothesis that the conditional probability of misreporting (under-report and over-reporting) is the same given the demographic group x. This calculation is further described in Appendix 2.

Once we have established the correlation between the misreporting error and the covariate, we will use the data to calculate the parameters needed to calculate the bias of estimates. These parameters, listed below, include the probability of receipt given demographic group, and the conditional probability of misreporting errors given demographic group and receipt status (overreporting/under-reporting rates).

$$
\begin{aligned}
\pi_{1} & =P(y=1 \mid x=1) \text { is the probability of true food stamp receipt given } x=1 \\
\pi_{0} & =P(y=1 \mid x=0) \text { is the probability of true food stamp receipt given } x=0 \\
r_{11} & =P\left(y^{\prime}=0 \mid y=1 \& x=1\right) \text { probability of under-reporting given receipt and } \mathrm{x}=1 \\
r_{10} & =P\left(y^{\prime}=0 \mid y=1 \& x=0\right) \text { probability of under-reporting given receipt and } \mathrm{x}=0 \\
r_{01} & =P\left(y^{\prime}=1 \mid y=0 \& x=1\right) \text { probability of overr-reporting given no receipt and } \mathrm{x}=1 \\
r_{00} & =P\left(y^{\prime}=1 \mid y=0 \& x=0\right) \text { probability of overr-reporting given no receipt and } \mathrm{x}=0
\end{aligned}
$$

These parameters will calculate the bias when we estimate a univariate linear model of reported food stamp receipt on the covariate of interest. With the estimate of the model from reported data,

$$
y_{i}^{\prime}=\alpha^{*}+x_{i} \beta^{*}+\eta_{i}
$$

and the true model from administrative data,

$$
y_{i}=\alpha+x_{i} \beta+\epsilon_{i}
$$

the bias is calculated as

$$
\beta^{*}-\beta=\left(\left(1-\pi_{1}\right) r_{01}-\pi_{1} r_{11}\right)-\left(\left(1-\pi_{0}\right) r_{00}-\pi_{0} r_{10}\right) .
$$

For each covariate, if the misreporting error is correlated with the covariate, we will also explore how the data corresponds with the two cases of minimized bias in the presence of misreporting. We will assess broadly whether the misreporting probability meets criteria 1 and 2 , and if the criteria are met, how well the parameters fit the sub-conditions (1.1)-(1.2) and (2.1)-(2.2) for minimized bias. For ease of comprehension, we will define a few terms to describe various differences we will measure to understand the bias.

For the first sub-condition of Case 1 (1.1), we will refer to the difference between the total false positive probability of demographic group $x=0$ and the total false positive probability of demographic group $x=1$ as Total False Positive Difference.

$$
\left(1-\pi_{1}\right) r_{01}-\left(1-\pi_{0}\right) r_{00}
$$


For the second sub-condition of Case 1 (1.2), we will refer to the difference between the total false negative probability of demographic group $\mathrm{x}=0$ and the total false negative probability of demographic group $\mathrm{x}=1$ as Total False Negative Difference.

$$
\pi_{1} r_{11}-\pi_{0} r_{10}
$$

The bias can be calculated as the difference between total false positive difference and total false negative difference.

$$
\left(\left(1-\pi_{1}\right) r_{01}-\left(1-\pi_{0}\right) r_{00}\right)-\left(\pi_{1} r_{11}-\pi_{0} r_{10}\right)
$$

For the first and second sub-condition of Case 2 (2.1 and 2.2) and, we will refer to the difference between the total false negative probability and the total false positive probability of a demographic group as Total Misreporting Contrast of [Demographic Group].

Total Misreporting Contrast of Demographic Group x=1:(1- $\left.\pi_{1}\right) r_{01}-\pi_{1} r_{11}$

Total Misreporting Contrast of Demographic Group x=0: $\left(1-\pi_{0}\right) r_{00}-\pi_{0} r_{10}$

The bias can be calculated as the difference between total misreporting contrasts of $x=1$ and $x=0$ demographic groups.

$$
\beta^{*}-\beta=\left(\left(1-\pi_{1}\right) r_{01}-\pi_{1} r_{11}\right)-\left(\left(1-\pi_{0}\right) r_{00}-\pi_{0} r_{10}\right)
$$

Each of the demographic groups will also have the following abbreviated labels:

\begin{tabular}{|lcc|}
\hline Covariate & Demographic Group & Label \\
\hline Income & Below Poverty Line & Poor \\
& Above Povery Line & Non-poor \\
\hline Household Type & Single Parent Household & SPH \\
& Non-Single Parent Household & Non-SPH \\
\hline Age of Household Head & Above or Equal to Age 50 & Elderly \\
& Below Age 50 & Non-elderly \\
\hline Race & White & White \\
& Nonwhite & Non-white \\
\hline
\end{tabular}

\section{$5.1 \quad$ Income}

The income variable $\mathrm{x} 1$ is defined as

$$
x 1_{i}=\left\{\begin{array}{l}
1 \quad \text { if household income is below or equal the poverty line } \\
0 \quad \text { if household income is above the poverty line }
\end{array}\right.
$$

From the data given, we calculate each of the parameters below 


\begin{tabular}{|l|c|}
\hline Parameters & $\%$ \\
\hline$\pi_{1}=P(y=1 \mid x 1=1)$ & 22.2 \\
$\pi_{0}=P(y=1 \mid x 1=0)$ & 2.1 \\
$r_{11}=P\left(y^{\prime}=0 \mid y=1 \& x 1=1\right)$ & 14.2 \\
$r_{10}=P\left(y^{\prime}=0 \mid y=1 \& x 1=0\right)$ & 33.2 \\
$r_{01}=P\left(y^{\prime}=1 \mid y=0 \& x 1=1\right)$ & 14.0 \\
$r_{00}=P\left(y^{\prime}=1 \mid y=0 \& x 1=0\right)$ & 1.3 \\
\hline$\beta^{*}$ & 27.2 \\
$\beta$ & 20.0 \\
\hline Bias of Estimate: $\beta^{*}-\beta$ & 7.2 \\
\hline
\end{tabular}

The administrative data show the poor have a much higher likelihood of being on food stamps than the nonpoor and the true estimate is $\beta=\pi_{1}-\pi_{0}=20 \%$. Both the poor and the nonpoor have high under-reporting rates of $14.2 \%$ and $33.2 \%$, respectively. The over-reporting rate, on the other hand, is much higher for the poor than the nonpoor with rates of $14.0 \%$ and $1.3 \%$, respectively.

Based on the $\chi^{2}$ test, estimated at 39.9 for false negatives and 793.9 for false positives on 1 degree of freedom, both types of misreporting errors are significantly correlated with $\mathrm{x} 1$. The overall bias $\beta^{*}-\beta$ is calculated at $7.2 \%$. If misreporting is not correlated with covariates, the bias would be $(\beta)\left(-\nu_{01}-\nu_{10}\right)=-5.0 \%$. We look at the factors that contribute the size of the current bias.

There are two criteria that are preconditions for the bias to be minimized in the presence of a large correlated misreporting.

The income covariates satisfy criterion 1, which requires the demographic group more likely to adopt food stamps be more likely to over-report and less likely to under-report. The poor is much more likely to adopt food stamps than the nonpoor and based on $r_{11}<r_{10}$ and $r_{01}>r_{00}$, the poor is also more likely to over-report and less likely to under-report. Merely satisfying one of these criteria does not preclude large biases, but these criteria are necessary in order for small bias to exist. We will explore how the sub-conditions (1.1) and (1.2) are met given that the initial criterion is satisfied.

Sub-condition 1.1: Total false positive difference between the poor and the nonpoor $\left(1-\pi_{0}\right) r_{00}-(1-$ $\left.\pi_{1}\right) r_{01}$ has to be minimized. In the sensitivity Appendix Table A.1, we measure how the conditional probabilities of over-reporting $r_{00}$ and $r_{01}$ affect the total false positive difference. Because $\pi_{1}>$ $\pi_{0} \Rightarrow\left(1-\pi_{1}\right)<\left(1-\pi_{0}\right)$, the region where sub-condition 1.1 is fulfilled and the difference is minimized lies in the region where $r_{01}>r_{00}$. With low participation rate low for both demographic groups, the total false positive difference is extremely sensitive to any variation between overreporting rates between the two groups. The false positive difference seems to be minimized only when the over-reporting is slightly greater for the poor and the difference between over-reporting rates is quite low $r_{01} \approx r_{00}$. This happens either when the over-reporting rates are not correlated with the demographic groups or when both over-reporting rates are low. However, because the over-reporting rate for the poor is much higher than that for the nonpoor, the total false positive difference is also quite significant at $9.7 \%$.

Sub-condition 1.2: Total false negative difference between the poor and the nonpoor $\pi_{0} r_{10}-\pi_{1} r_{11}$ has to be minimized. In the Appendix Table A.2, we measure how the under-reporting rates $r_{10}$ and 
$r_{11}$ affect the total false negative difference. The under-reporting parameters lie in the region that fulfills criterions 1 with greater under-reporting for nonpoor than poor $r_{11}<r_{10}$. From current parameters, the total false negative difference is calculated at $2.5 \%$. The sensitivity table makes apparent that under-reporting has less of an impact on estimate bias when participation rate is low. With participation rates for poor and nonpoor at $22.2 \%$ and $2.1 \%$, respectively, the total false negative difference remain under $14 \%$ even in the most extreme scenario.

The income covariate partially satisfies Criterion 2, which requires that a demographic group with low participation rate (below 50\%) be more likely to under-report than over-report. With the income variable, we find that both the poor and the nonpoor both have relatively low participation rate, so for the bias to be minimized they both have to be more likely to under-report than to over-report. Whereas the nonpoor roughly fulfills this criterion with $r_{10}>\pi_{00}$, the poor is on the cusp with $r_{11} \approx r_{01}$. We will explore how the sub-conditions (2.1) and (2.2) are met given that the data only partially satisfy the initial criterion.

Sub-condition 2.1: Total misreporting contrast of the poor $\left(1-\pi_{1}\right) r_{01}-\pi_{1} r_{11}$ has to be minimized. In Appendix Table A.3, we measure how the over-reporting rate $r_{01}$ and the under-reporting rate $r_{11}$ affect total misreporting contrast of the poor. The parameters lie within the boundary of the region that fulfills criterion 2 . The boundary is where under-reporting is equal to over-reporting $r_{11} \approx r_{01}$. Echoing the generalization made by Bound et al, when participation rate is low, the bias would be small only if over-reporting is much lower than under-reporting. However, because for the poor, the over-reporting rate is similar to the under-reporting, the total misreporting contrast of the poor contributes significantly to the bias in the estimates. From the existing parameters, the total misreporting contrast of the poor is calculated at $7.8 \%$.

Sub-condition 2.2: Total misreporting contrast of the nonpoor $\left(1-\pi_{0}\right) r_{00}-\pi_{0} r_{10}$ has to be minimized. In sensitivity Table A.4, we measure how the over-reporting rate $r_{00}$ and the under-reporting rate $r_{10}$ affect total misreporting contrast of the nonpoor. The parameters for the nonpoor lie in the region where criterion 2 is fulfilled, with under-reporting greater than over-reporting. Because the over-reporting rate of the nonpoor is much lower than under-reporting rates, the total misreporting contrast is at $0.5 \%$ with almost no impact on the bias.

We can look at how the bias would change if all the criteria were fulfilled by the data, such that the poor, who are more likely to be on food stamps than the nonpoor, are more likely to over-report and less likely to under-report than the nonpoor. And both the poor and the nonpoor, with low participation rates, are more likely to under-report than to over-report. Our sensitivity table shows that even if under-reporting reach rates as high as 60\% (holding parameters not being sensitized constant), the bias would not exceed $10 \%$. Overall, our sensitivity shows that the bias would not exceed $16 \%$.

Summary: The data for the income covariate indicates that, with low participation rates, overreporting rates have a much more visible impact on bias than under-reporting. Because the poor has a non-negligible over-reporting rate, the bias in the estimate can grow large. On the other hand, under-reporting has a much more limited impact on the bias and as long as the relationship between under-reporting of the poor and the nonpoor fulfills criterion 1, the bias created from under-reporting would be low. Overall, the general behavior of the groups, fulfills the following criteria

1. low participation rate and a lower probability of over-reporting than under-reporting for the 
nonpoor,

2. the greater probability of the demographic group more likely to be on food stamps (poor) to over-report and

3. the greater probability of the demographic group less likely to be on food stamps (nonpoor) to under-report,

The last criterion - greater probability of nonpoor to under-report - has less of impact on bias then the first two. The absolute value of the bias is higher than what it would be if misreporting rates are not correlated with the income demographic groups. The bias would be further minimized if, given the low participation rates, over-reporting was significantly lower than under-reporting for the poor.

\subsection{Household Type (Single Parent Households)}

The household type variable $\mathrm{x} 2$ is defined as

$$
x 2_{i}=\left\{\begin{array}{l}
1 \quad \text { if single parent household } \\
0 \quad \text { if otherwise }
\end{array}\right.
$$

From the data given, we calculate each of the parameters below

\begin{tabular}{|l|c|}
\hline Parameters & $\%$ \\
\hline$\pi_{1}=P(y=1 \mid x 2=1)$ & 20.0 \\
$\pi_{0}=P(y=1 \mid x 2=0)$ & 2.5 \\
$r_{11}=P\left(y^{\prime}=0 \mid y=1 \& x 2=1\right)$ & 22.3 \\
$r_{10}=P\left(y^{\prime}=0 \mid y=1 \& x 2=0\right)$ & 23.3 \\
$r_{01}=P\left(y^{\prime}=1 \mid y=0 \& x 2=1\right)$ & 12.1 \\
$r_{00}=P\left(y^{\prime}=1 \mid y=0 \& x 2=0\right)$ & 1.4 \\
\hline$\beta^{*}$ & 22.0 \\
$\beta$ & 17.6 \\
\hline Bias of Estimate: $\beta^{*}-\beta$ & 4.4 \\
\hline
\end{tabular}

The administrative data show the single parent households (SPH) have a much higher likelihood of being on food stamps than other households (nonSPH) and the true estimate is $\beta=\pi_{1}-\pi_{0}=17.6 \%$. Both SPH and nonSPH have high under-reporting rates of $22.3 \%$ and $23.3 \%$, respectively. The overreporting, on the other hand, is higher for SPH than the nonSPH with rates of $12.4 \%$ and $1.4 \%$, respectively.

Based on the $\chi^{2}$ calculated off the contingency table, which is estimated at 0.13 for false negatives and 591.3 for false positives with 1 degree of freedom, under-reporting errors are not correlated and over-reporting errors are significantly correlated with $\mathrm{x} 2$. The bias $\beta^{*}-\beta$ is calculated at $4.4 \%$. If the misreporting errors are not correlated with the covariates, the bias would be $-4.4 \%$.

We will explore Criteria 1 and 2 for the household type covariate. 
The household type covariates partially satisfies criterion 1, which requires the demographic group more likely to adopt food stamps (SPH) be more likely to over-report and less likely to underreport. The parameters show that the SPH is more likely to over-report than nonSPH $r_{01}>r_{00}$, but the under-reporting rates between the two groups are quite close and not significantly correlated with the covariate. Therefore, criterion 1 is only partially fulfilled. We will explore how the subconditions (1.1) and (1.2) are met given that the data does not fully satisfy the initial criterion.

Sub-condition 1.1: Appendix Table B.1 measures how the over-reporting rates $r_{00}$ and $r_{01}$ affect the total false positive difference $\left(1-\pi_{0}\right) r_{00}-\left(1-\pi_{1}\right) r_{01}$. The over-reporting parameters $r_{00}$ and $r_{01}$ for the SPH covariate lie in the region where criterion 1 is fulfilled region and SPH over-reporting rate is higher than nonSPH over-reporting rate. Because of low participation rates, over-reporting has significant impact on bias. The total false positive difference is very sensitive to differences between the over-reporting rates. The difference is minimized when, aside from fulfilling criterion 1, the difference between the two over-reporting rates are small. This would be true if the over-reporting rates are not correlated with the demographic groups or the rates are low for both demographic groups. The total false positive difference is calculated at $8.3 \%$ from existing parameters. This rate and the bias would be further diminished if over-reporting rate for SPH decreases without violating criterion 1.

Sub-condition 1.2: Appendix Table B.2 measures how the under-reporting rates $r_{10}$ and $r_{11}$ affect the total false negative difference $\pi_{0} r_{10}-\pi_{1} r_{11}$. Because under-reporting rates according to our $\chi^{2}$ test is not correlated with the demographic groups, under-reporting parameters $r_{10}$ and $r_{11}$ lies on the boundary where $r_{11} \approx r_{10}$. The rates do not fulfill criterion 1 , but we find that the Total false negative difference actually has less of an impact on the bias than the total false positive difference. With under-reporting uncorrelated with the covariates and $r_{11} \approx r_{10}$, the total false negative difference remain under $11 \%$ even in the most extreme scenario. The total false negative difference is calculated at $3.9 \%$ from existing parameters.

The household type covariate satisfies Criterion 2, which requires that the demographic groups with low participation rate be more likely to under-report than over-report. With the household type variable, we find that both the SPH $\left(\pi_{1}=20 \%\right)$ and the nonSPH $\left(\pi_{0}=2.5 \%\right)$ have participation rates below $50 \%$, and both fulfill criterion 2. We will explore how the sub-conditions (2.1) and (2.2) are met given the initial criterion is satisfied.

Sub-condition 2.1 Appendix Table B.3 measures how the over-reporting rate $r_{01}$ and the underreporting rate $r_{11}$ of single parent households affect total misreporting contrast $\left(1-\pi_{1}\right) r_{01}-\pi_{1} r_{11}$. The parameters lie in the region fulfilling criterion 2, where under-reporting is greater than overreporting. Although the initial criterion is fulfilled, the Total misreporting contrast of SPH is still quite significant at $5.2 \%$. The total misreporting contrast would be minimized if over-reporting of $\mathrm{SPH}$ decreases further relative to the under-reporting for SPH.

Sub-condition 2.2: Appendix Table B.4 measures how the over-reporting rate $r_{00}$ and the underreporting rate $r_{10}$ of non-single parent households affect total misreporting contrast $\left(1-\pi_{0}\right) r_{00}-$ $\pi_{0} r_{10}$. The parameters lie in the region that satisfies criterion 2 where under-reporting exceeds over-reporting. The misreporting parameters of the nonSPH for the household type covariate lie in this region. Due to the low over-reporting rate relative to the under-reporting, we find the total misreporting contrast is quite low at $0.8 \%$. 
We can look at the range of the bias if both criteria were fulfilled by the data, such that single parent households, who are more likely to be on food stamps, are more likely to over-report and less likely to under-report than the nonSPH. And both the SPH and the nonSPH, with low participation rates, have lower over-reporting rate than under-reporting rate. In this case, since under-reporting is not correlated with the demographic groups, the under-reporting rates between demographic groups would stay roughly equal. Our sensitivity table shows that even if the under-reporting reach rates as high as $60 \%$ (holding other parameters constant), the bias would not exceed $6.0 \%$. Overall, our sensitivity shows that the bias would not exceed $14 \%$.

Summary: The data for household type demographic groups indicate under-reporting is not significantly correlated with the covariate, while over-reporting is correlated. The parameters and sensitivity tables further support the conjecture that, with low participation rates, over-reporting rates have a much more visible impact on bias than under-reporting. The bias was limited because the data fulfilled these criteria,

1. low participation rate and a lower probability of over-reporting than under-reporting for both $\mathrm{SPH}$ and nonSPH,

2. the greater probability of the demographic group more likely to be on food stamps (SPH) to over-report

We find the bias for Household Type covariate to be lower than Income covariate, partly because both SPH and nonSPH fulfill criterion 2 with lower over-reporting rate than under-reporting. The absolute value of the bias is the same as what it would be if the misreporting errors are not correlated with the covariate. The bias would be further reduced if over-reporting for SPH decreases, within the bounds of criterion 1 .

\subsection{Elderly Households}

The elderly household variable $\mathrm{x} 3$ is defined as

$$
x 3_{i}=\left\{\begin{array}{l}
1 \quad \text { if head of household is above or equal to age } 50 \\
0 \quad \text { if head of household is below age } 50
\end{array}\right.
$$

From the data given, we calculate each of the parameters below

\begin{tabular}{|l|c|}
\hline Parameters & $\%$ \\
\hline$\pi_{1}=P(y=1 \mid x 3=1)$ & 3.3 \\
$\pi_{0}=P(y=1 \mid x 3=0)$ & 5.1 \\
$r_{11}=P\left(y^{\prime}=0 \mid y=1 \& x 3=1\right)$ & 21.5 \\
$r_{10}=P\left(y^{\prime}=0 \mid y=1 \& x 3=0\right)$ & 23.7 \\
$r_{01}=P\left(y^{\prime}=1 \mid y=0 \& x 3=1\right)$ & 1.6 \\
$r_{00}=P\left(y^{\prime}=1 \mid y=0 \& x 3=0\right)$ & 3.1 \\
\hline$\beta^{*}$ & -2.7 \\
$\beta$ & 3.1 \\
\hline Bias of Estimate: $\beta^{*}-\beta$ & -0.9 \\
\hline
\end{tabular}


The administrative data show the elderly households have a similar probability of being on food stamps as nonelderly households and the true estimate is $\beta=\pi_{1}-\pi_{0}=-1.8 \%$. The difference in food stamp receipt between demographic groups is quite low. Both elderly and nonelderly have similar under-reporting rates of, $21.5 \%$ and $23.7 \%$, respectively. The over-reporting for both are also quite low, with rates of, $1.6 \%$ and $3.1 \%$, respectively.

Based on the $\chi^{2}$ calculated off the contingency table, which is estimated at 0.54 for false negatives and 36.1 for false positives with 1 degree of freedom, over-reporting errors are significantly correlated with $\mathrm{x} 3$ and under-reporting errors are not correlated. If neither of the misreporting errors is correlated with the covariate, then the bias would shrink the coefficient such that $\beta^{*}-\beta=\left(\pi_{1}-\right.$ $\left.\pi_{0}\right)\left(-\nu_{01}-\nu_{10}\right)=-1.8 \%+0.45 \%=-1.35 \%$. Instead, the correlated errors inflate the coefficient, exaggerating the difference in food stamp receipt between demographic groups. The uncorrelated bias is $0.45 \%$, slightly lower than the current bias of $-0.9 \%$.

We will explore the Criterion 1 and 2 for the household type covariate.

Since the participation rate for each demographic group is quite close with nonelderly slightly more likely than elderly to be on food stamps, and $\beta=\pi_{1}-\pi_{0}=-1.8 \%$ is not much higher than 0 , criterion 1 requires over-reporting rates for demographic groups to be close to equality with overreporting for nonelderly slightly greater than over-reporting for the elderly. The household age covariate fulfills this criteria for over-reporting. Criterion 1 also requires under-reporting rates for demographic groups to be close to equality with under-reporting for the elderly slightly greater than under-reporting for the nonelderly. Since under-reporting is not significantly correlated with the age demographic group, the parameters are quite close to the boundary of equality. The parameters do not fulfill criterion 1 for under-reporting.

Given criterion 1 is partially fulfilled, Appendix Table C.1 and C.2 measure how the parameters affect the total false positive difference and the total false negative difference. We see that, because of the low participation rates and correlation with the covariate, over-reporting has a greater impact on bias than under-reporting. Currently, the total false positive difference is $1.4 \%$, as compared to the total false negative difference of $0.5 \%$. If over-reporting rate for the non-elderly was decreased (without falling below over-reporting rate for the elderly), we would have lower total false positive difference and eliminate most of the biases. Overall, because participation rates are quite close between the demographic groups, the bias generated is also quite low.

Criterion 2 requires over-reporting rates for demographic groups to be well below under-reporting rates if participation rates are low. Since participation rates are at $3.3 \%$ and $5.1 \%$ for elderly and nonelderly, and both groups have much lower over-reporting than under-reporting, this criterion is fulfilled.

Appendix Table C.3 and C.4 measures how the parameters affect the total misreporting contrast for elderly and nonelderly, respectively. They show that that over-reporting has a greater impact on bias due to the very low participation rates in both groups. Appendix Table C.4 shows that if over-reporting rate for the non-elderly was decreased (without falling below over-reporting rate for the elderly), we would have lower total misreporting contrast for the nonelderly and eliminate most of the remaining biases. The total misreporting contrast is calculated at $0.8 \%$ for elderly and $1.7 \%$ for the nonelderly. 
We can look at the range of the bias if both criteria were fulfilled by the data, such that the nonelderly, who are more likely to be on food stamps, are more likely to over-report. And both the elderly and the nonelderly, with low participation rates, have much lower over-reporting rate than under-reporting rate. Our sensitivity tables show that if we keep over-reporting rates low (at current rates) and assume under-reporting is not correlated with the demographic groups such that $r_{11} \approx r_{10}$, even if the under-reporting rates reach rates as high as $60 \%$, the bias would not exceed $2 \%$. On the other hand, if over-reporting increased significantly, even when we fulfill these criteria, the bias could be as high as $20 \%$ so that the estimate $\beta^{*}$ could be as low as $-21 \%$, as compare to the true coefficient $-0.9 \%$.

Summary: When the participation rates for both demographic groups are quite low and the estimate $\pi_{1}-\pi_{0}$ is close to 0 , under-reporting has a marginal impact on bias. This is especially true since under-reporting for the elderly is found to be uncorrelated with the demographic groups. Over-reporting has a much greater impact on bias. The higher over-reporting for nonelderly results in $0.9 \%$ bias whereas bias would be $0.45 \%$ if misreporting errors are not correlated. The bias would be diminished if the over-reporting rates for both demographic groups decrease, with the overreporting rate for the nonelderly slightly higher than the over-rate for elderly (based on criterion 1). If over-reporting is increased for either of the demographic groups such that the rates are significantly different between demographic groups, the bias could be dramatically increased and the coefficient on elderly households significantly inflated.

\subsection{Race (White and Nonwhite)}

The race variable $\mathrm{x} 4$ is defined as

$$
x 4_{i}= \begin{cases}1 & \text { if household is white } \\ 0 & \text { if household is nonwhite }\end{cases}
$$

From the data given, we calculate each of the parameters below

\begin{tabular}{|l|c|}
\hline Parameters & $\%$ \\
\hline$\pi_{1}=P(y=1 \mid x 4=1)$ & 1.9 \\
$\pi_{0}=P(y=1 \mid x 4=0)$ & 14.4 \\
$r_{11}=P\left(y^{\prime}=0 \mid y=1 \& x 4=1\right)$ & 20.7 \\
$r_{10}=P\left(y^{\prime}=0 \mid y=1 \& x 4=0\right)$ & 24.0 \\
$r_{01}=P\left(y^{\prime}=1 \mid y=0 \& x 4=1\right)$ & 1.4 \\
$r_{00}=P\left(y^{\prime}=1 \mid y=0 \& x 4=0\right)$ & 7.1 \\
\hline$\beta^{*}$ & -14.2 \\
$\beta$ & 12.5 \\
\hline Bias of Estimate: $\beta^{*}-\beta$ & -1.7 \\
\hline
\end{tabular}

The administrative data show nonwhite households have higher probability of being on food stamps than white households and the true estimate is $\beta=\pi_{1}-\pi_{0}=-12.5 \%$. The under-reporting rates are slightly lower for whites than nonwhite, but they are close, with rates of $20.7 \%$ and $24 \%$, respectively. The over-reporting is slightly lower for whites than nonwhite, with rates of, $1.4 \%$ and $7.1 \%$, respectively. 
Based on the $\chi^{2}$ estimated at 1.18 for false negatives and 309.2 for false positives with 1 degree of freedom, over-reporting errors are significantly correlated with $\mathrm{x} 4$. The bias is quite low considering the amount of misreporting and the value of the true coefficient. It is calculated at $\beta^{*}-\beta=-1.7 \%$. If misreporting errors are uncorrelated with the covariate, the bias would be $3.2 \%$.

We will explore Criterion 1 and 2 for the race covariate.

The race covariate partially satisfies Criterion 1, which requires the demographic group more likely to adopt food stamps (nonwhite) be more likely to over-report and less likely to under-report. Nonwhite demographic group, which is more likely to adopt food stamps is also more likely to over-report than whites, but they are also slightly more likely to under-report than nonwhites. We will explore how the sub-conditions (1.1) and (1.2) are met given that the data does not fully satisfy the initial criterion.

Sub-condition (1.1): Appendix Table D.1 measures how the over-reporting rates $r_{00}$ and $r_{01}$ affect the total false positive difference $\left(1-\pi_{0}\right) r_{00}-\left(1-\pi_{1}\right) r_{01}$. The table shows that the over-reporting parameters fall within the region fulfilling criterion 1, such that over-reporting rates of white is greater than non-white. The false positive difference is extremely sensitive to over-reporting rates and much like other covariates, false positive difference is minimized when the difference between the over-reporting rates are quite small $r_{00} \approx r_{01}$. Since over-reporting is correlated with covariate, minimized false positive difference is more likely to occur where both over-reporting rates for both demographic groups are low. The higher over-reporting rate for nonwhite of $7.1 \%$ creates a significant false positive difference of $4.8 \%$.

Sub-condition (1.2): Appendix Table D.2 measure how the under-reporting rates $r_{10}$ and $r_{11}$ affect the total false negative difference $\pi_{0} r_{10}-\pi_{1} r_{11}$. The under-reporting parameters fall on the boundary where $r_{11} \approx r_{10}$, outside of the region where this criterion 1 is fulfilled. Therefore, the false negative difference is not negligible at around $3.6 \%$.

The race covariate satisfies criterion 2, which requires a demographic group with low participation rates to have lower over-reporting rates than under-reporting rates. Both white and nonwhite have low participation rates, $1.9 \%$ and $14.4 \%$ respectively, and are far less likely to over-report than to under-report. We will explore how the sub-conditions (2.1) and (2.2) are met given this initial criterion is satisfied.

Sub-condition (2.1): Appendix Table D.3 measures how the over-reporting rate $r_{01}$ and the underreporting rate $r_{11}$ of white households affect total misreporting contrast $\left(1-\pi_{1}\right) r_{0} 1-\pi_{1} r_{1} 1$. White, which has a very low participation rate, also has a significantly lower over-reporting rate than under-reporting rates. Consequently, the total misreporting contrast is quite low at around $1.0 \%$.

Sub-condition (2.2): Appendix Table D.4 measures how the over-reporting rate $r_{00}$ and the underreporting rate $r_{10}$ of nonwhite households affect total misreporting contrast $\left(1-\pi_{0}\right) r_{00}-\pi_{0} r_{10}$. Nonwhite has a low participation rate, but one higher than white. It also has a significant overreporting rate. However, because the over-reporting rate is much lower than the under-reporting rate, the misreporting contrast for nonwhite is relatively low at $2.6 \%$, resulting in a lower overall bias in the estimate. 
Summary: The bias is lower than what it would have been if the misreporting errors are not correlated with the covariate. Overall, we find under-reporting is not correlated with the demographic groups but over-reporting is correlated. Due to the participation rates, over-reporting has a more significant impact on the bias. Because criterion 2 is fulfilled with much lower over-reporting than under-reporting for both demographic groups, we find that bias for race is quite low.

\subsection{Multivariate Model}

Appendix Table E shows the linear regression of each variable in a univariate model and multivariate models. We find that the bias for the data remains relatively similar across the models. In fact, the absolute value of the bias is slightly reduced from the univariate model when we combine the data into a multivariate model. Because of correlation between misreporting errors and over-reporting, the bias generally acts to inflate the coefficient of the variables. Elderly household is an exception with a slightly significant variable in the administrative data model rendered insignificant in the reported model. This variable is also the only coefficient which switched signs when added to the combined model. While the signs were switched, the value of the elderly coefficient bias is preserved. When we look at the bias relative to the true coefficients, we find the bias ratio to coefficients stay relatively constant or decreases for significant variables.

Monte carlo simulation of the variable and the food stamps data show the absolute value of the average simulated bias shifting towards the point of no bias when we adopt the combined model rather than the simple model. Appendix F shows the histogram of simulation results. This result is not conclusive for all model estimates as it is due to the positive correlation between covariates. If the covariates are not correlated or negatively correlated, the bias would not be diminished in the combined model.

However, we can conclude that the bias in the multivariate model is generally the same sign and similar in size as the univariate model and the standard deviation of the simulated bias is similar between the univariate and the multivariate models.

\section{Conclusions}

Under-reporting tends to play a smaller role in creating the bias than over-reporting. Underreporting is generally not correlated with the covariate, and have less of impact on estimate bias due to low true participation rates.

While under-reporting rates tend not to be correlated, over-reporting rates are always correlated with the covariates. Over-reporting, which is attributed to retrieval error caused by inconsistent participation in food stamps or confusion with other government programs, is much more likely to occur for demographic groups that are more likely to be on food stamps and other subsidy programs. Under-reporting on the other may be more commonly attributed to non-reporting, when an individual leaves an item blank, which may be equally likely for different demographic groups. This is only true for data not imputed, as over-reporting and under-reporting patterns can 
be quite different after missing values have been imputed.

For the food stamps data, participation estimates tend to have lower bias if two criteria are fulfilled: 1) low over-reporting for all demographic groups and lower over-reporting rates relative to underreporting 2) greater likelihood of the demographic group more likely to participate in food stamps to over-report. Of less importance, is a third factor which is that the demographic group less likely to participate in food stamps be more likely to under-report. The last factor is less relevant since participation rates are low (below 50\%) across all demographic groups and under-reporting is generally uncorrelated with the covariates.

We find the first criterion generally to be true. There is lower over-reporting than under-reporting for all demographic groups, however the over-reporting rates are not always negligible, sometimes reaching as high as $12 \%$. The second criterion is true for all covariates. The demographic group more likely to be on food stamps is also more likely to over-report.

Due to above factors, we find the bias on participation estimates to be limited. When covariates are combined into one multivariate model, size of bias relative to coefficients remain relatively stable or diminishes for highly significant coefficients. Based on these findings, we believe the food stamp participation models using reported census data can still have reasonable estimates despite high misreporting rates.

The analysis in this paper was based on simple models that assume a linear relationship between food stamp receipt probabilities and covariates. The relationship may be more accurately described by a logit model where log ratios of probabilities describe the nonlinear relationship between food stamp receipt and covariates. We believe that some of the conclusions from this paper can apply in the logit model, but because the relationship is more complex, it would have to be furthered evaluated before we can make this conclusion. 


\section{References}

[1] Bishop, John A., John Formby, and Lester Zeager (1996). The Impact of Food Stamps on US Poverty in the 1980s: A Marginal Dominance Analysis. Economica, 63:250, S141-S162.

[2] Bollinger, Christopher and Martin David (1997). Modeling Discrete Choice with Response Error: Food Stamp Participation. Journal of the American Statistical Association, 92 (439), pp. 827-835.

[3] Bound, John, Charles Brown and Nancy Mathiowetz (2001). Measurement Error in Survey Data. Handbooks of Econometrics. Volume 5, ed. By J.J. Hackman and E. Learner. Elsevier: Amsterdam.

[4] Cunnyham, Karen E., Laura Castner, and allen Schirm (2008). Reaching Those in Need: State Food Stamp Participation Rates in 2006. Alexandria, VA: U.S. Department of Agriculture, Food and Nutrition Service.

[5] Hausman, Jerry (2001). "Mismeasured variable in Econometric Analysis: Problems from the Right and Problems from the Left. The Journal of Econometric Perspectives, Vol. 15, No. 4, pp. 47-67.

[6] Hausman, Jerry, Jason Abrevaya, F.M. Scott-Morton (1998). Misclassification Of The Dependent Variable In A Discrete-Response Setting. Journal of Econometrics, 87, 239-269.

[7] Jolliffe, Dean, Craig Gundersen, Laura Tiehen, and Joshua Winicki (2005). Food Stamp Benefits and Child Poverty American Journal of Agricultural Economics, 87(3): 569-581.

[8] Meyer, Bruce D., Wallace K.C. Mok, and James X. Sullivan. 2009 "The Under-Reporting of Transfers in Household Surveys: Its Nature and Consequences," NBER Working Paper No. 15181.

[9] Meyer, Bruce and Robert Goerge. 2011 Errors in Survey Reporting and Imputation and Their Effects on Estimates of Food Stamp Program Participation, NBER Working Paper.

[10] Moore Jeffrey C., Kent H. Marquis and Karen Bogen (1996). The SIPP Cognitive Research Evaluation Experiment: Basic Results and Documentation. The Survey of Income and Program Participation, Working Paper No. 212. Washington D.C.: U.S. Census Bureau.

[11] Wolkwitz, Kari (2008). Trends in Food Stamp Program Participation Rates: 2000 to 2006. Current Perspectives on Food Stamp Program Participation Series, Alexandria, VA: U.S. Department of Agriculture, Food and Nutrition Service.

[12] U.S. Department of Agriculture, Food and Nutrition Service. Characteristics of Supplemental Nutrition Assistance Program (SNAP) Households: Fiscal Year 2010

[13] U.S. Department of Agriculture, Food and Nutrition Service. Trends in Supplemental Nutrition Assistance Program Participation Rates: FY 2002 to FY 2009 


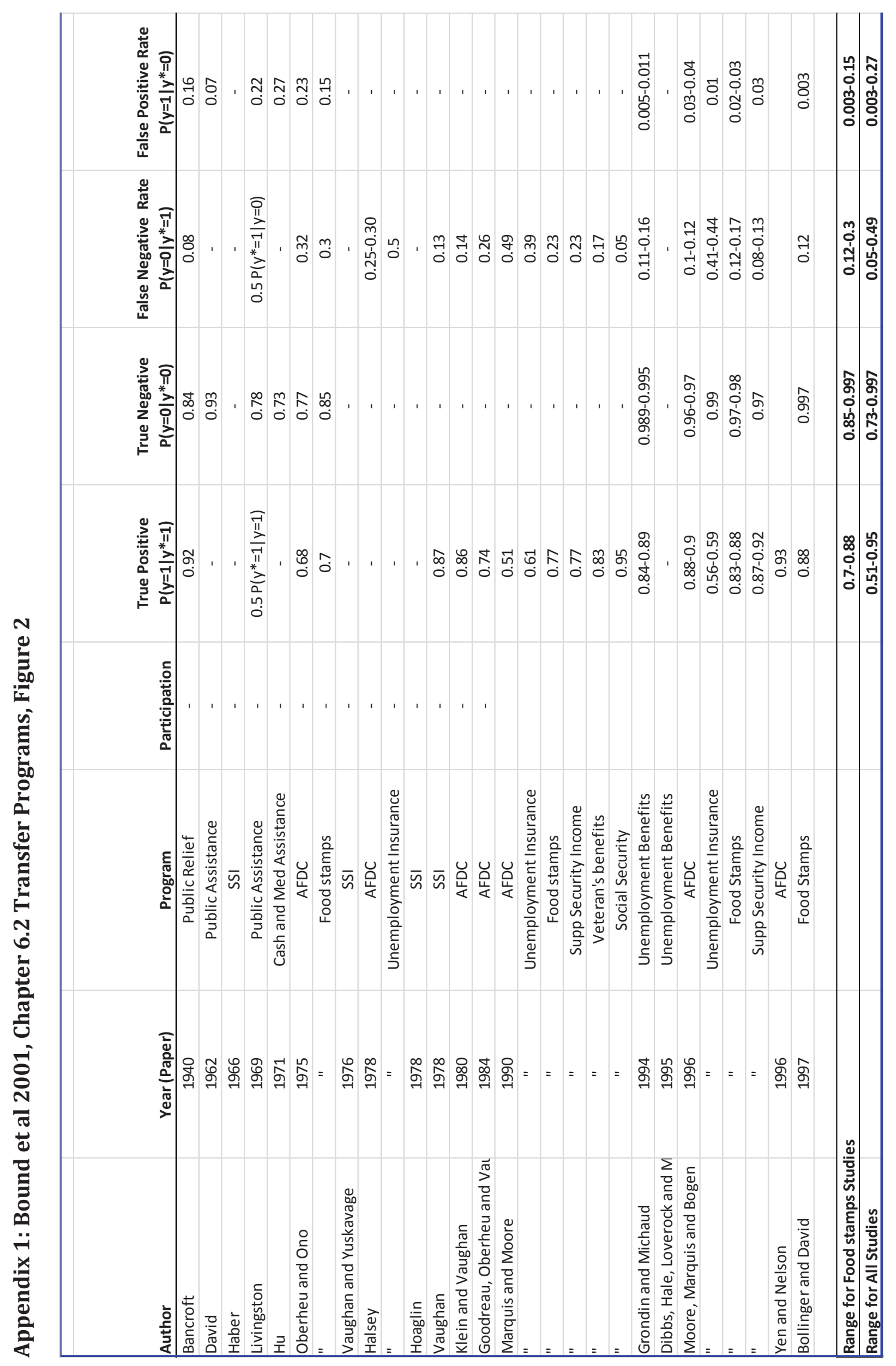


Appendix 2: How $\chi^{2}$ calculate to test significance of correlation between misreporting error (false negative or false positive) and covariate (demographic group)

Given the following cells

$$
\begin{aligned}
& m_{1}=\text { total samples size of } x=1 \\
& m_{0}=\text { total samples size of } x=0
\end{aligned}
$$

\begin{tabular}{|l|ll|l|}
\hline & $\mathrm{X}=1$ & $\mathrm{X}=0$ & \\
\hline $\mathrm{P}\left(\mathrm{Y}^{\prime}=1 \mid \mathrm{Y}=0\right)$ & $\mathrm{C}_{11}=\mathrm{r} 11^{*} \mathrm{p}(\mathrm{x}=1)$ & $\mathrm{C}_{21}=\mathrm{r} 10^{*} \mathrm{p}(\mathrm{x}=0)$ & $\mathrm{C}_{11}+\mathrm{C}_{21}$ \\
$\mathrm{P}\left(\mathrm{Y}^{\prime}=0 \mid \mathrm{Y}=0\right)$ & $\mathrm{C}_{12}=(1-\mathrm{r} 11)^{*} \mathrm{p}(\mathrm{x}=1)$ & $\mathrm{C}_{22}=(1-\mathrm{r} 10)^{*} \mathrm{p}(\mathrm{x}=0)$ & $\mathrm{C}_{12}+\mathrm{C}_{22}$ \\
\hline & $\mathrm{p}(\mathrm{x}=1)$ & $\mathrm{p}(\mathrm{x}=0)$ & $100 \%$ \\
\hline
\end{tabular}

To test null hypothesis: Over-reporting is not correlated with $\mathrm{X}$ (on 1 degree of freedom)

$$
\begin{gathered}
X^{2}=m_{1} \frac{\left(r_{11}-\left(C_{11}+C_{21}\right)\right)^{2}}{\left(C_{11}+C_{21}\right)}+m_{0} \frac{\left(r_{10}-\left(C_{11}+C_{21}\right)\right)^{2}}{\left(C_{11}+C_{21}\right)}+m_{1} \frac{\left(1-r_{11}-\left(C_{12}+C_{22}\right)\right)^{2}}{\left(C_{12}+C_{22}\right)}+m_{0} \frac{\left(1-r_{10}-\left(C_{12}+C_{22}\right)\right)^{2}}{\left(C_{12}+C_{22}\right)} \\
X^{2} \sim \chi_{d f=1}^{2}
\end{gathered}
$$

Given the following cells

\begin{tabular}{|l|ll|l|}
\hline & $\mathrm{X}=1$ & $\mathrm{X}=0$ & \\
\hline $\mathrm{P}\left(\mathrm{Y}^{\prime}=0 \mid \mathrm{Y}=1\right)$ & $\mathrm{D}_{11}=\mathrm{r}_{01} * \mathrm{p}(\mathrm{x}=1)$ & $\mathrm{D}_{21}=\mathrm{r}_{00} * \mathrm{p}(\mathrm{x}=0)$ & $\mathrm{D}_{11}+\mathrm{D}_{21}$ \\
$\mathrm{P}\left(\mathrm{Y}^{\prime}=1 \mid \mathrm{Y}=1\right)$ & $\mathrm{D}_{12}=\left(1-\mathrm{r}_{01}\right) * \mathrm{p}(\mathrm{x}=1)$ & $\mathrm{D}_{22}=\left(1-\mathrm{r}_{00}\right)^{*} \mathrm{p}(\mathrm{x}=0)$ & $\mathrm{D}_{12}+\mathrm{D}_{22}$ \\
\hline & $\mathrm{p}(\mathrm{x}=1)$ & $\mathrm{p}(\mathrm{x}=0)$ & $100 \%$ \\
\hline
\end{tabular}

To test null hypothesis: Under-reporting is not correlated with covariate

$$
\begin{gathered}
X^{2}=m_{1} \frac{\left(r_{01}-\left(D_{11}+D_{21}\right)\right)^{2}}{\left(D_{11}+D_{21}\right)}+m_{0} \frac{\left(r_{00}-\left(D_{11}+D_{21}\right)\right)^{2}}{\left(D_{11}+D_{21}\right)}+m_{1} \frac{\left(1-r_{01}-\left(D_{12}+D_{22}\right)\right)^{2}}{\left(D_{12}+D_{22}\right)}+m_{0} \frac{\left(1-r_{00}-\left(D_{12}+D_{22}\right)\right)^{2}}{\left(D_{12}+D_{22}\right)} \\
X^{2} \sim \chi^{2}{ }_{d f=1}
\end{gathered}
$$

For both tests, null hypothesis is rejected at the $5 \%$ level if $X^{2}>5.02$. The $X^{2}$ is based on the assumption that each of the counts are independent. However, due to repeated households in our sample, this value may be slightly inaccurate. For the purpose of our analysis, we use this test statistic as an approximate indicator of independence between misreporting error and covariate. 

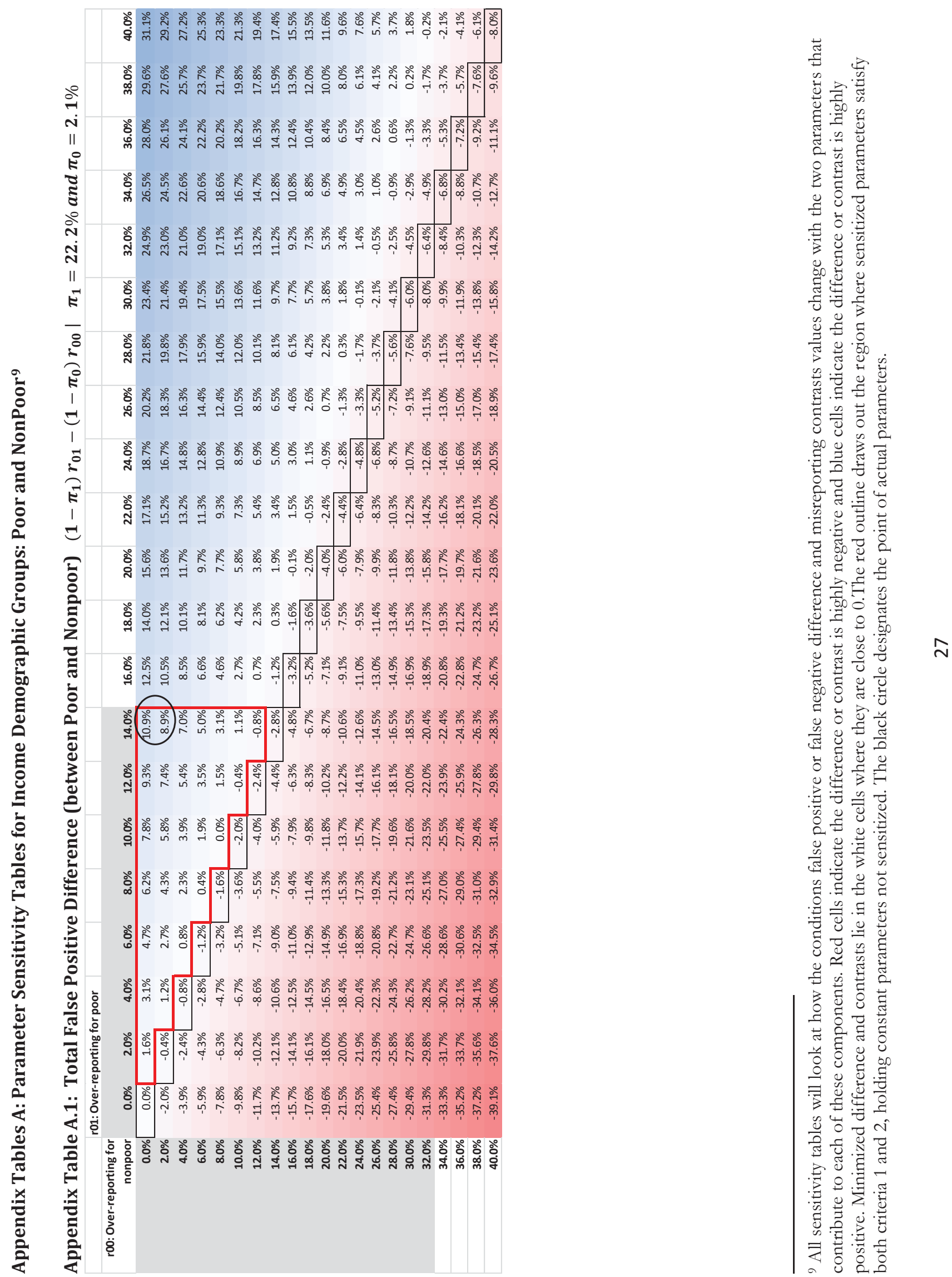


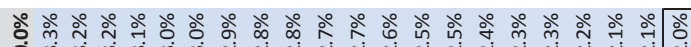

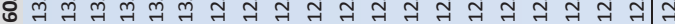

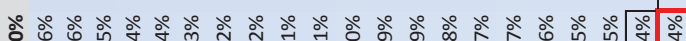

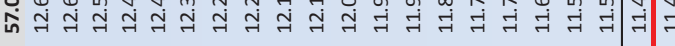

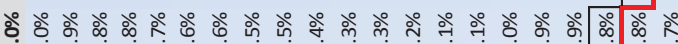

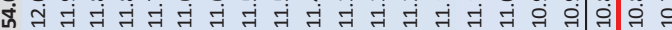

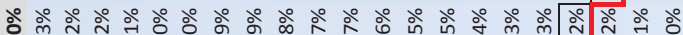

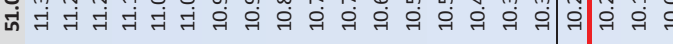

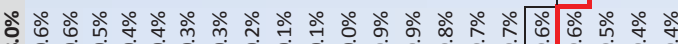

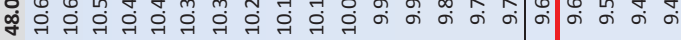

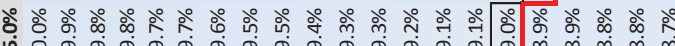
$\begin{array}{llllll} & & & \end{array}$

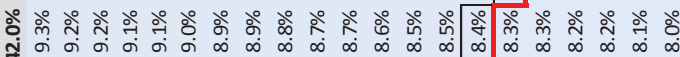

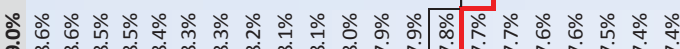

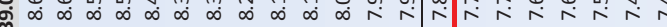

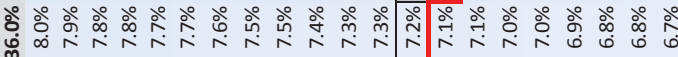

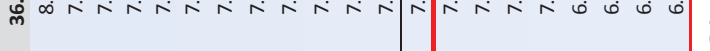

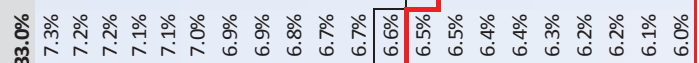

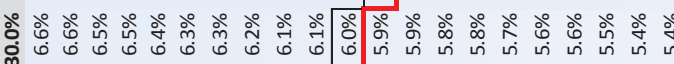

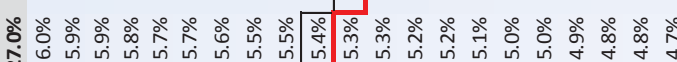

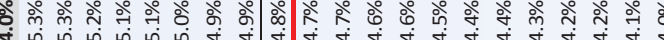

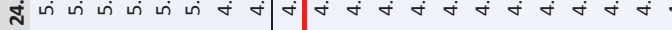

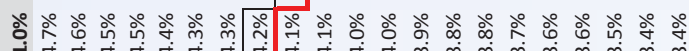

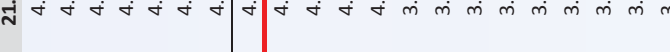

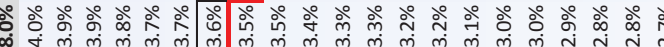

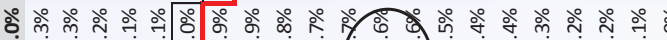

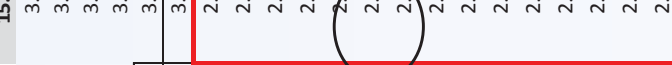

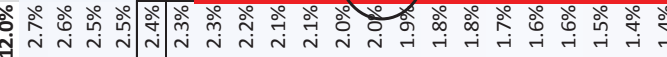

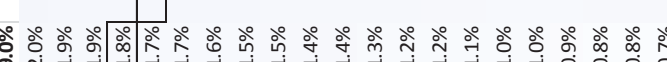

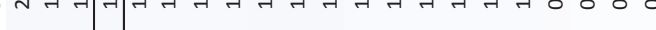

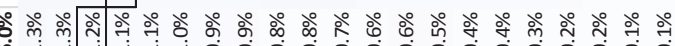

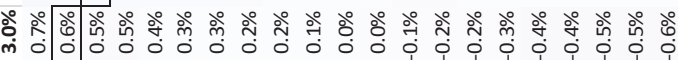

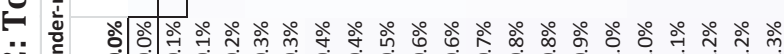
సุ $<\ddot{\exists}$

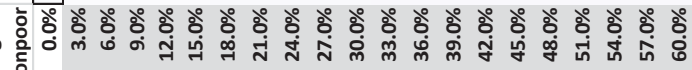
突 $\frac{\sqrt{2}}{\frac{2}{2}}$

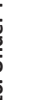
인

\section{萬}

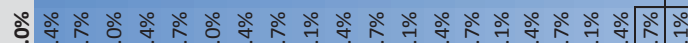

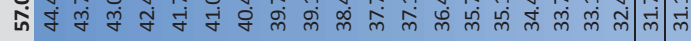

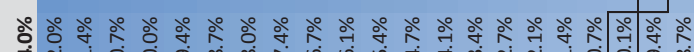

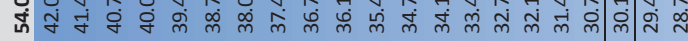

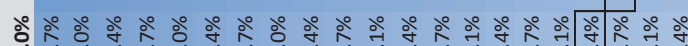
मีं में क्

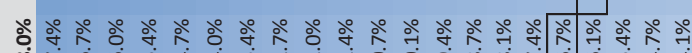
कें

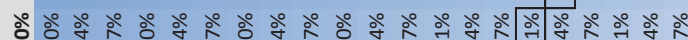

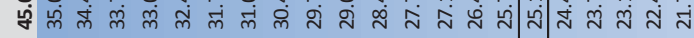

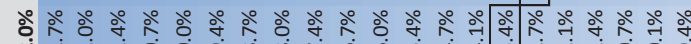
สู山m

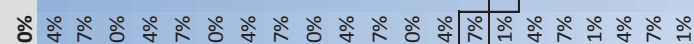
व்

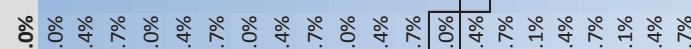
min

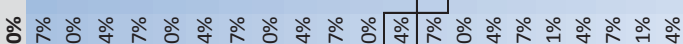

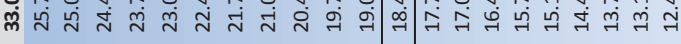

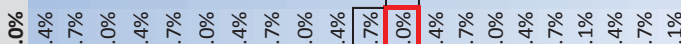

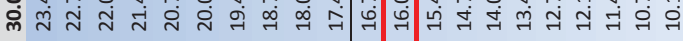

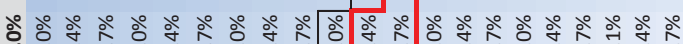
సं तं هं

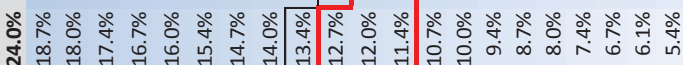
$\stackrel{\infty}{\sim}$

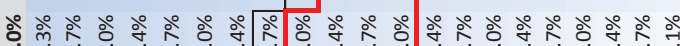

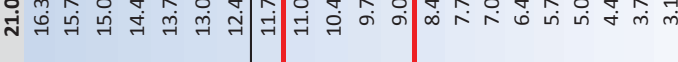

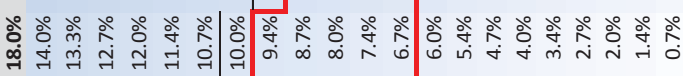

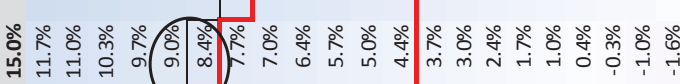
ம் नं नं की

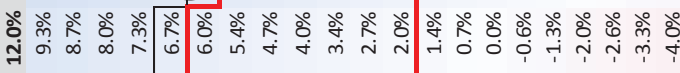

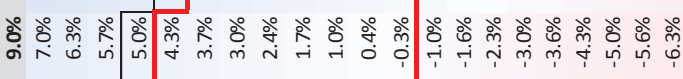

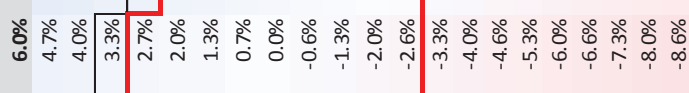

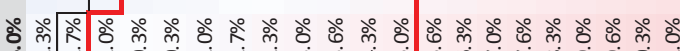

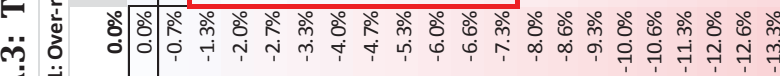
$<\ddot{\dot{d}}$

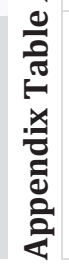

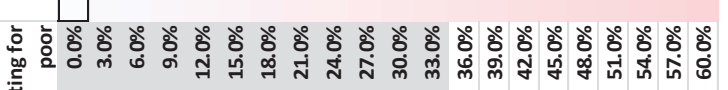




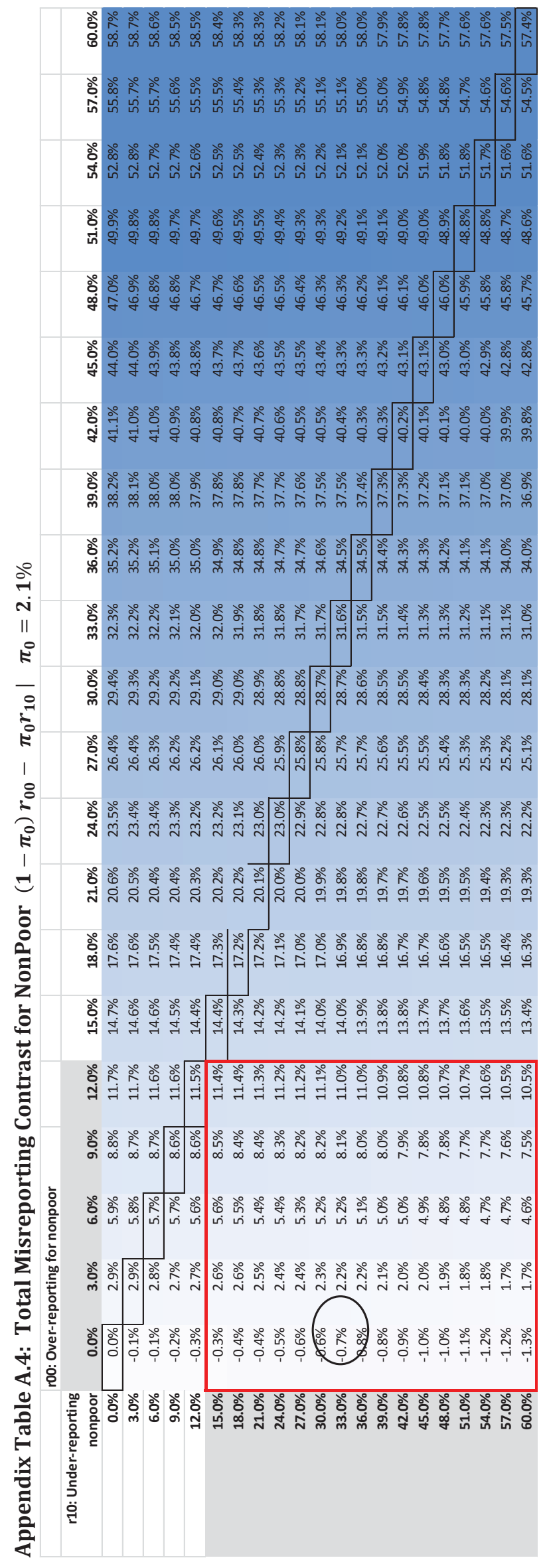



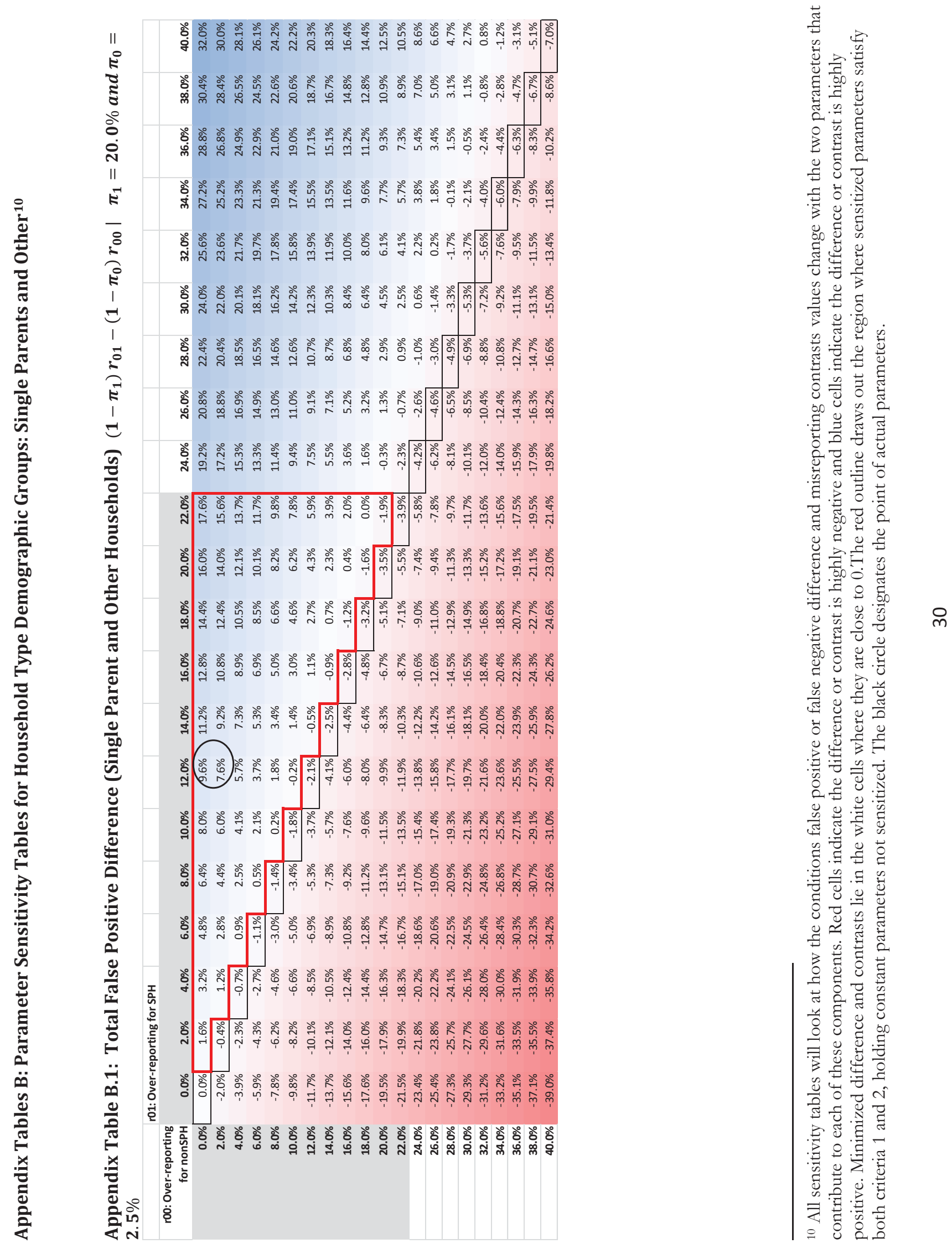


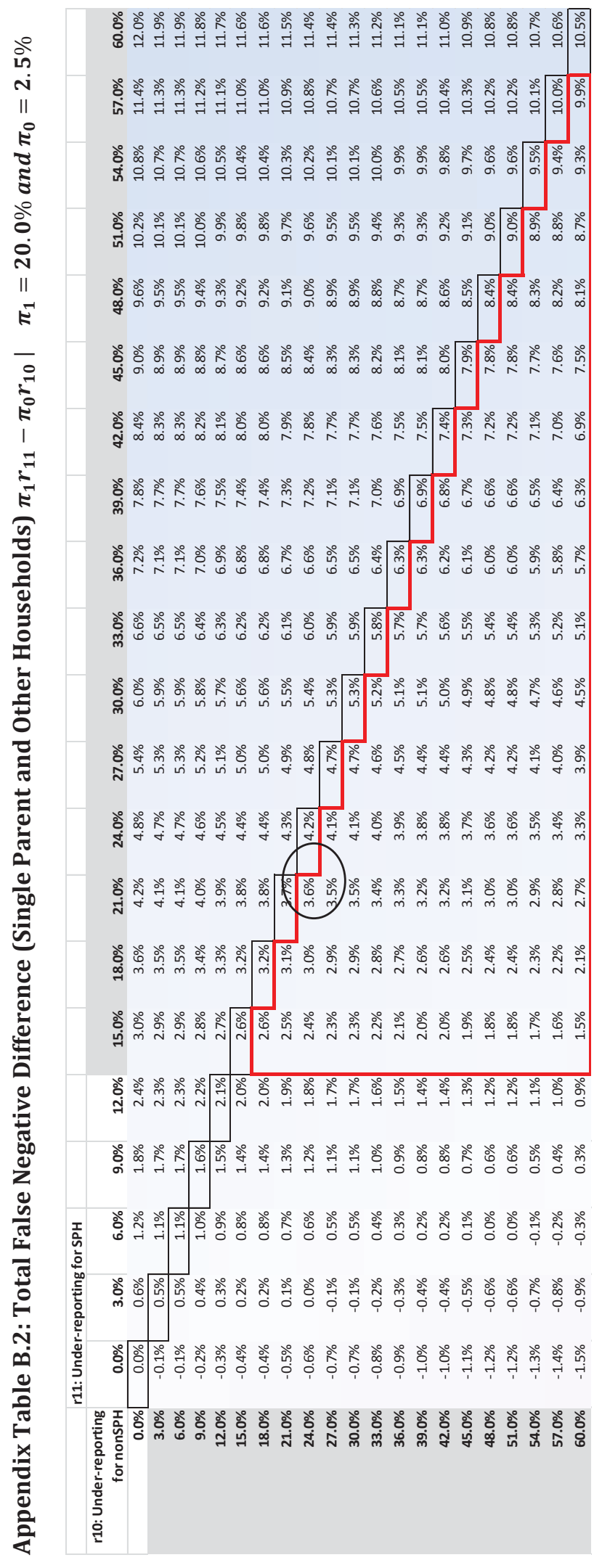



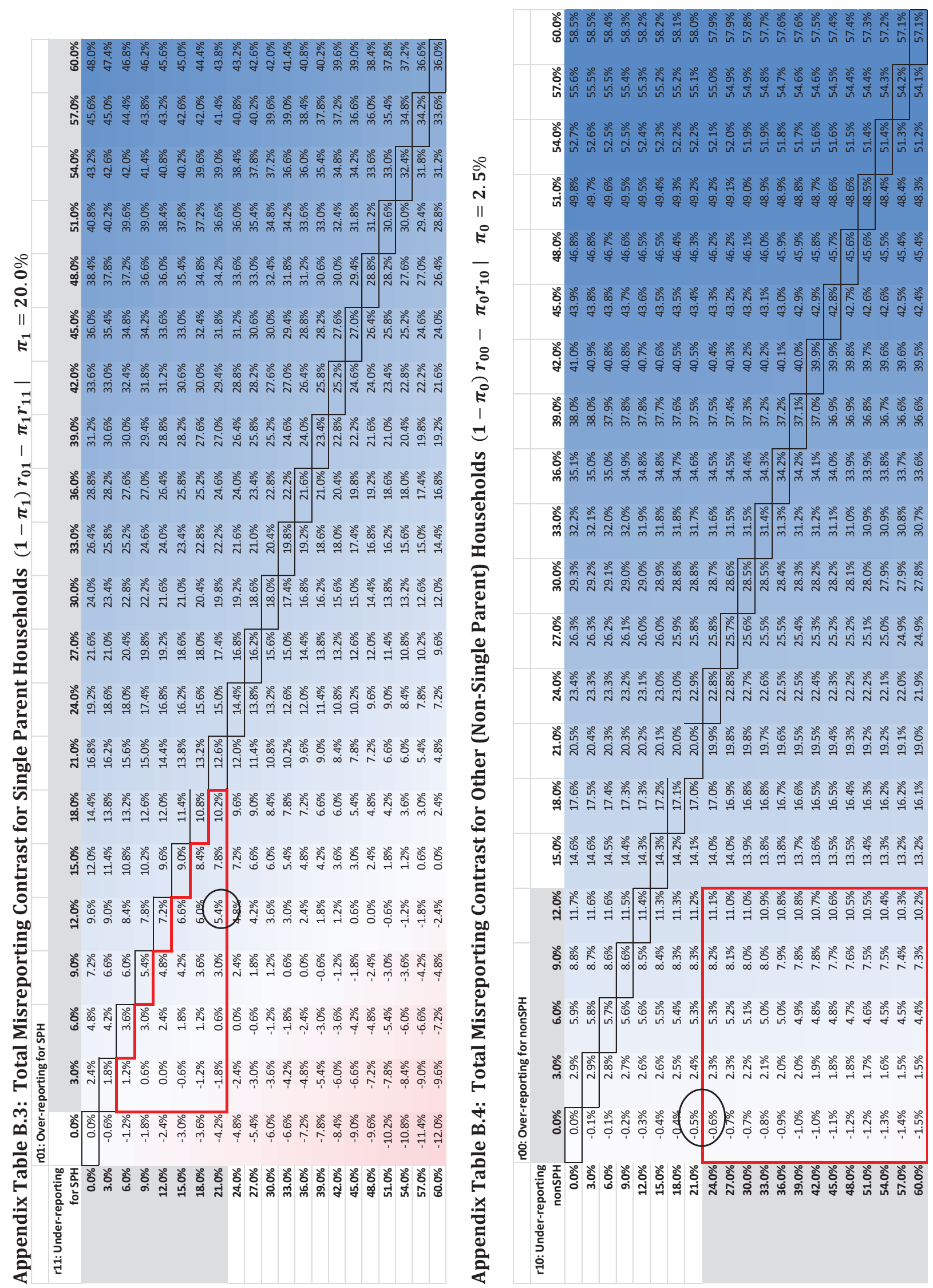


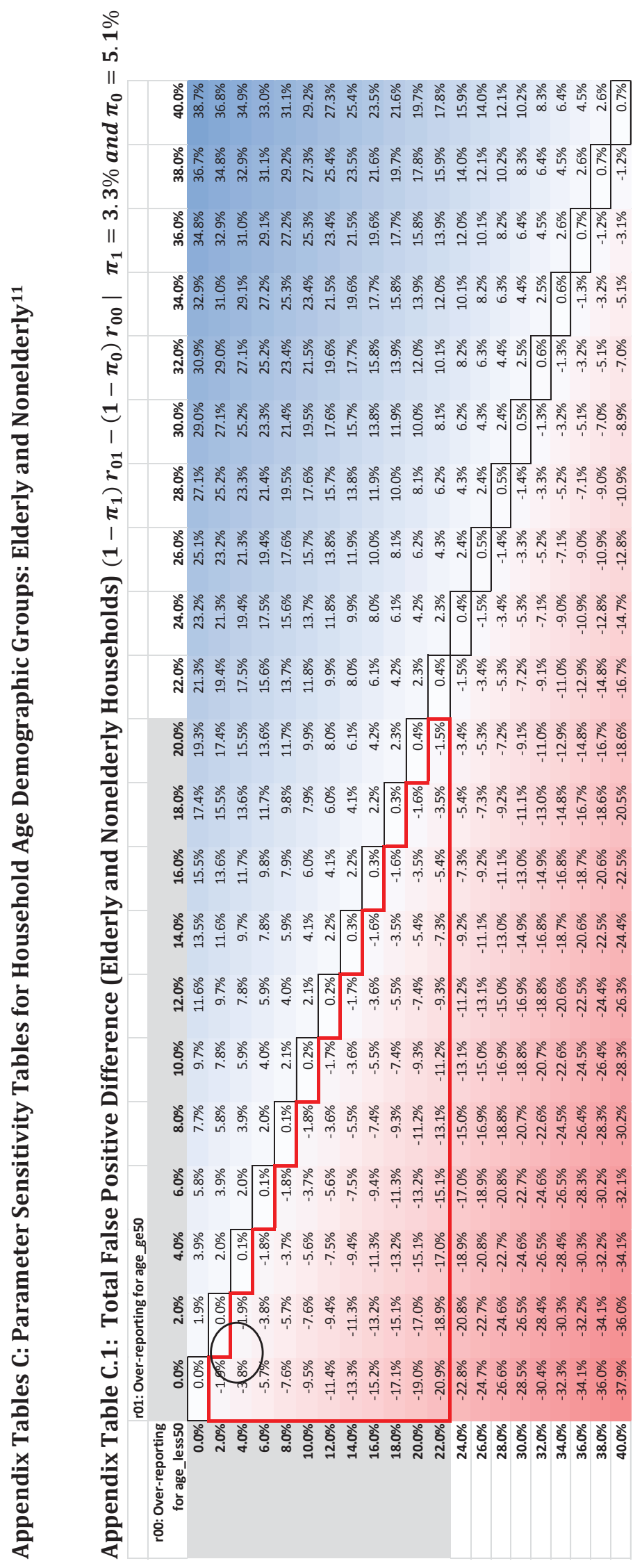

Iี

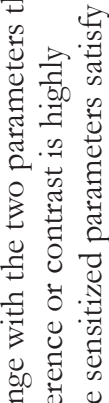
矛总离 荘 ह5 空 ठี so 0 o 氙焉

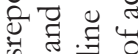
艺泀言 G ठั 击 矛造 0. $\stackrel{m}{m}$

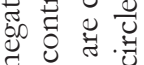
क मे 氖 ज मे 跑苛 $:=0$
0.0
0
0

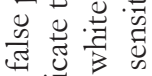

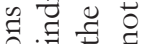
请 $\cong$ 등 0 . ฐ J 它

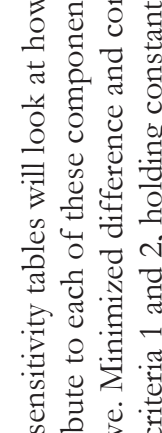

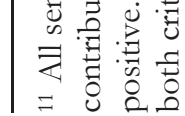



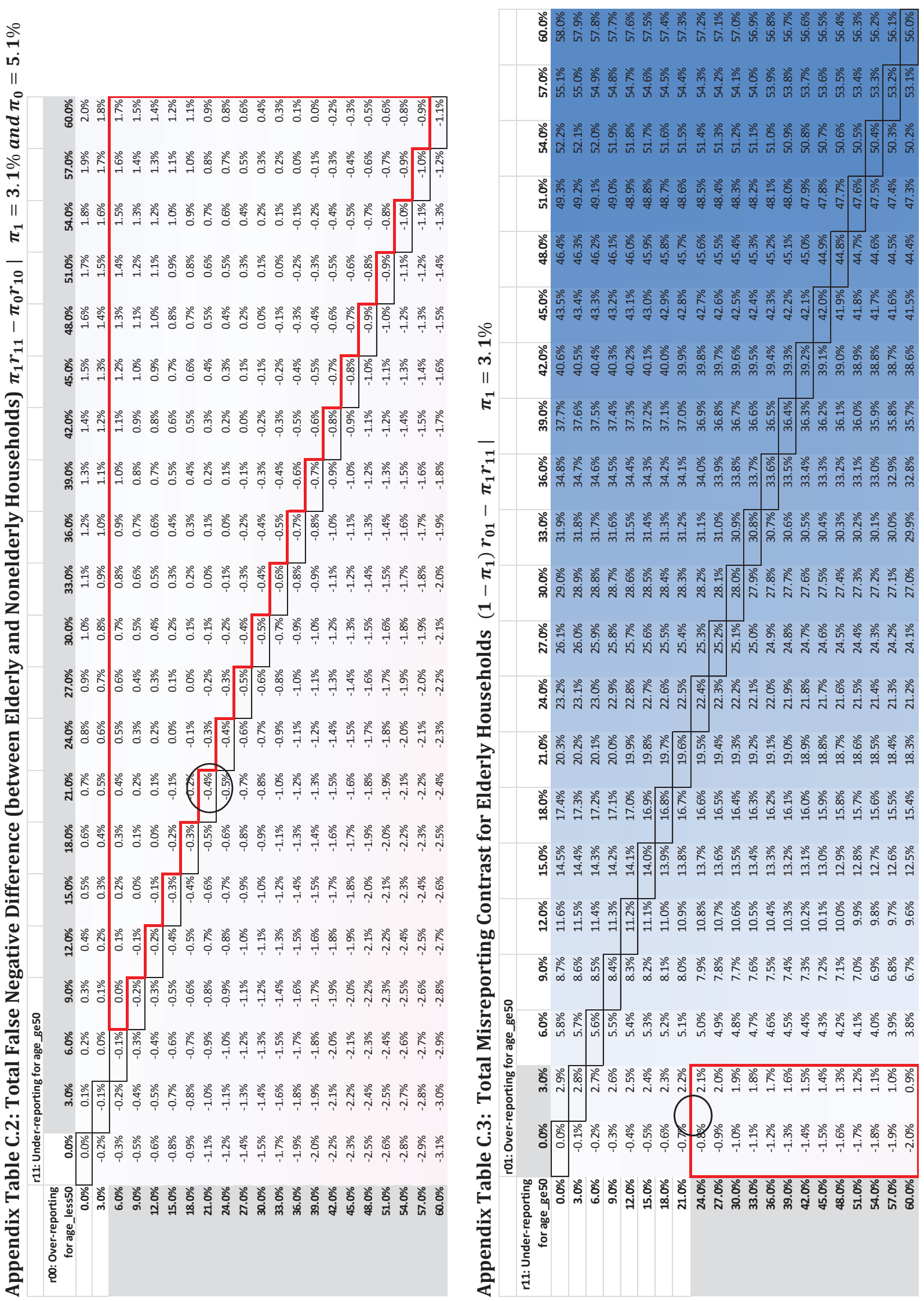


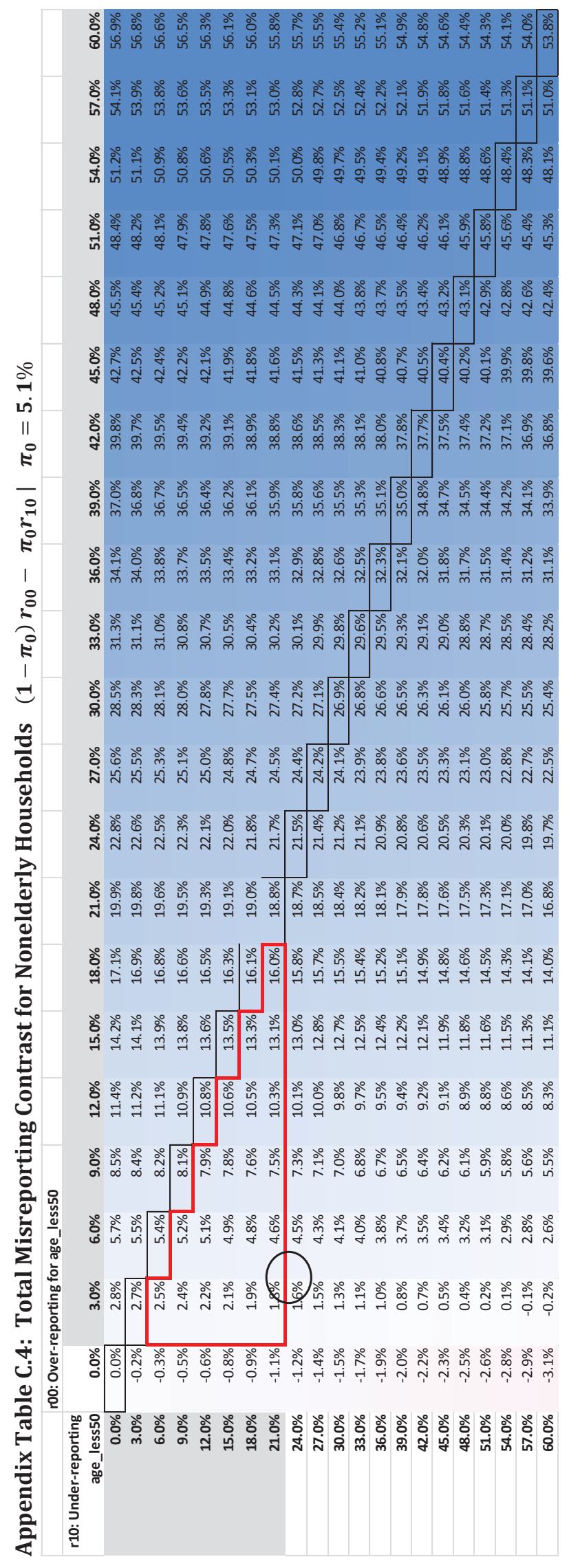



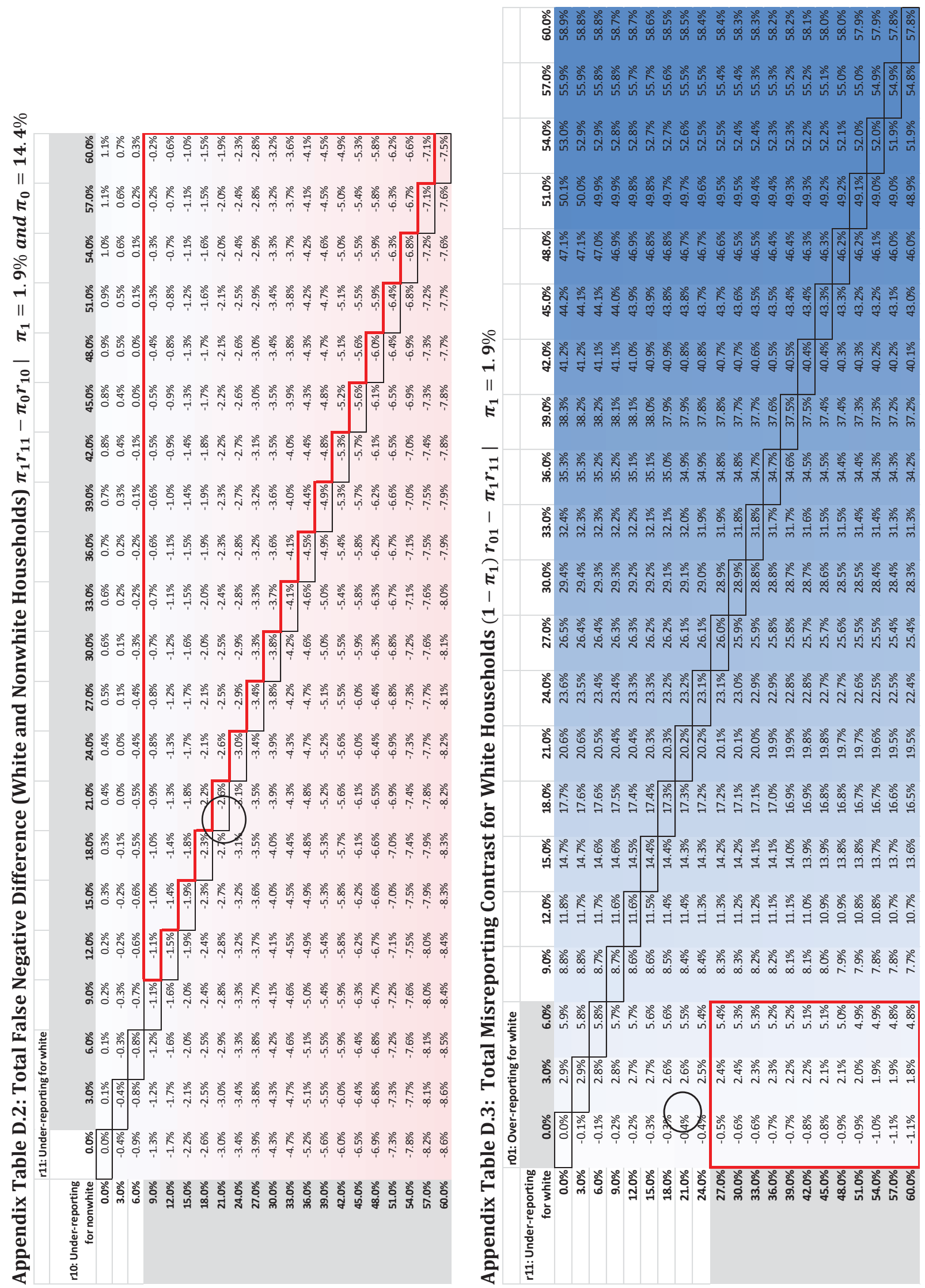


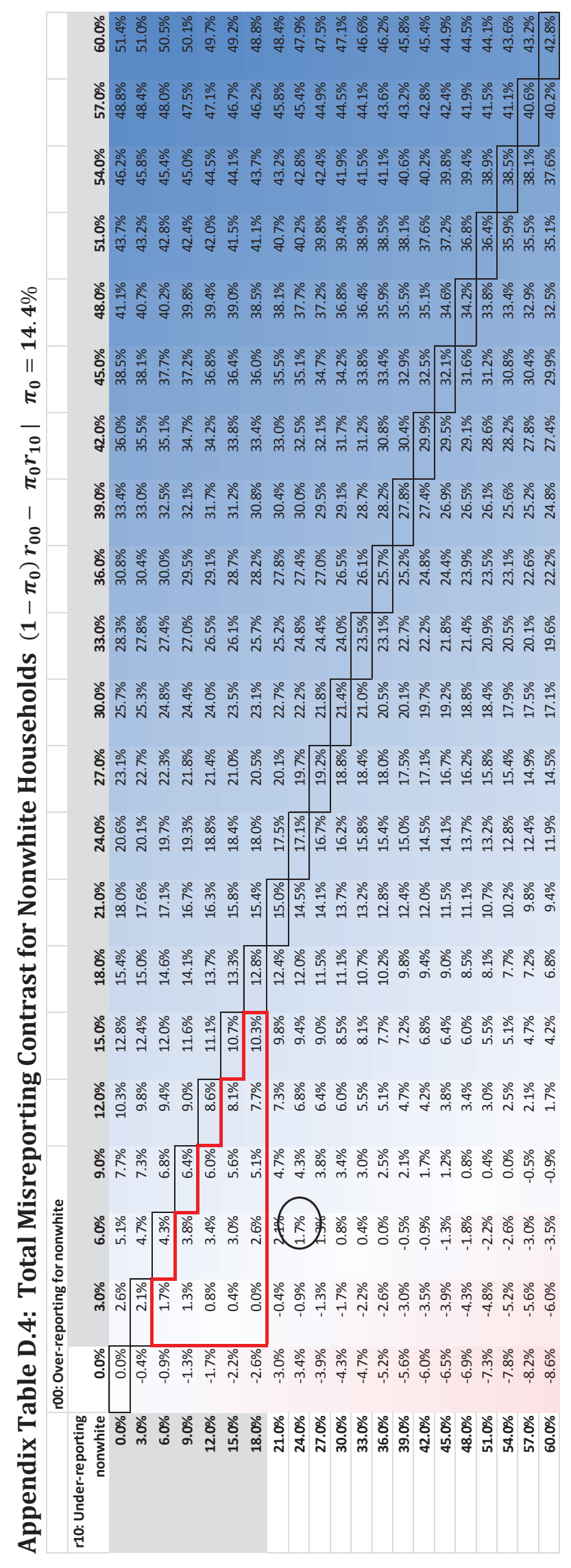




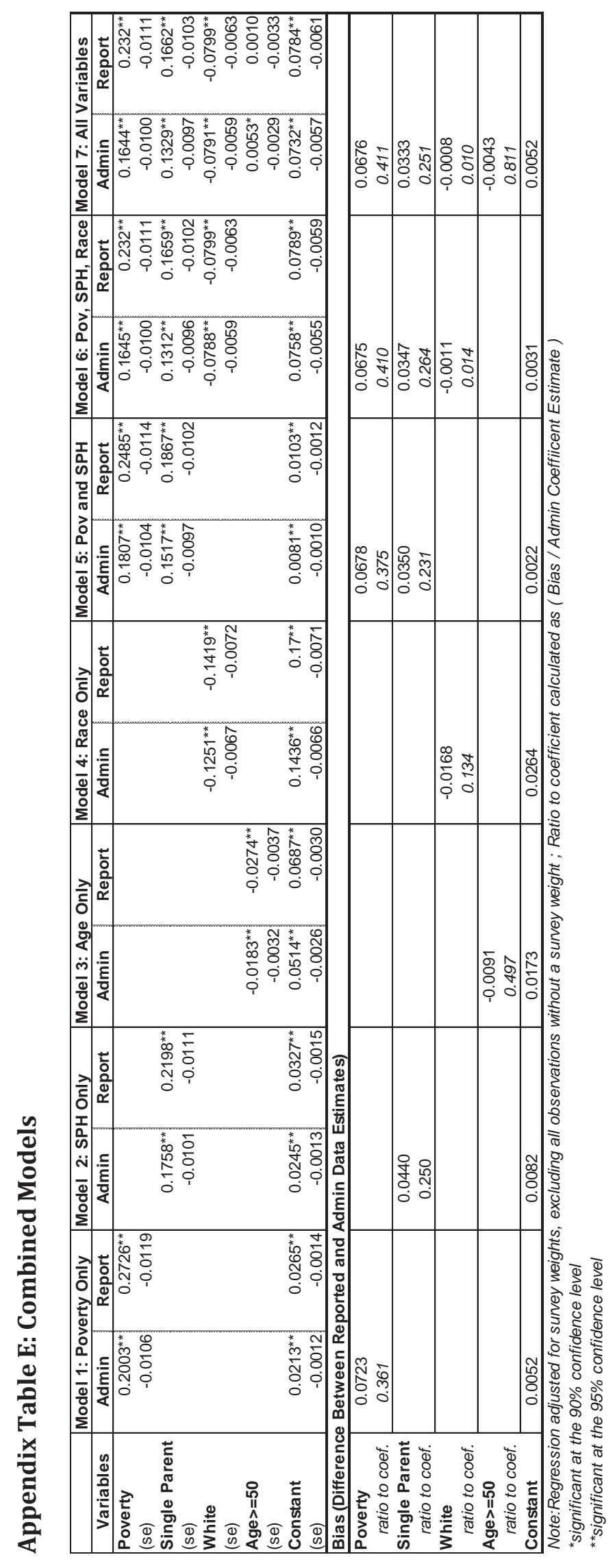




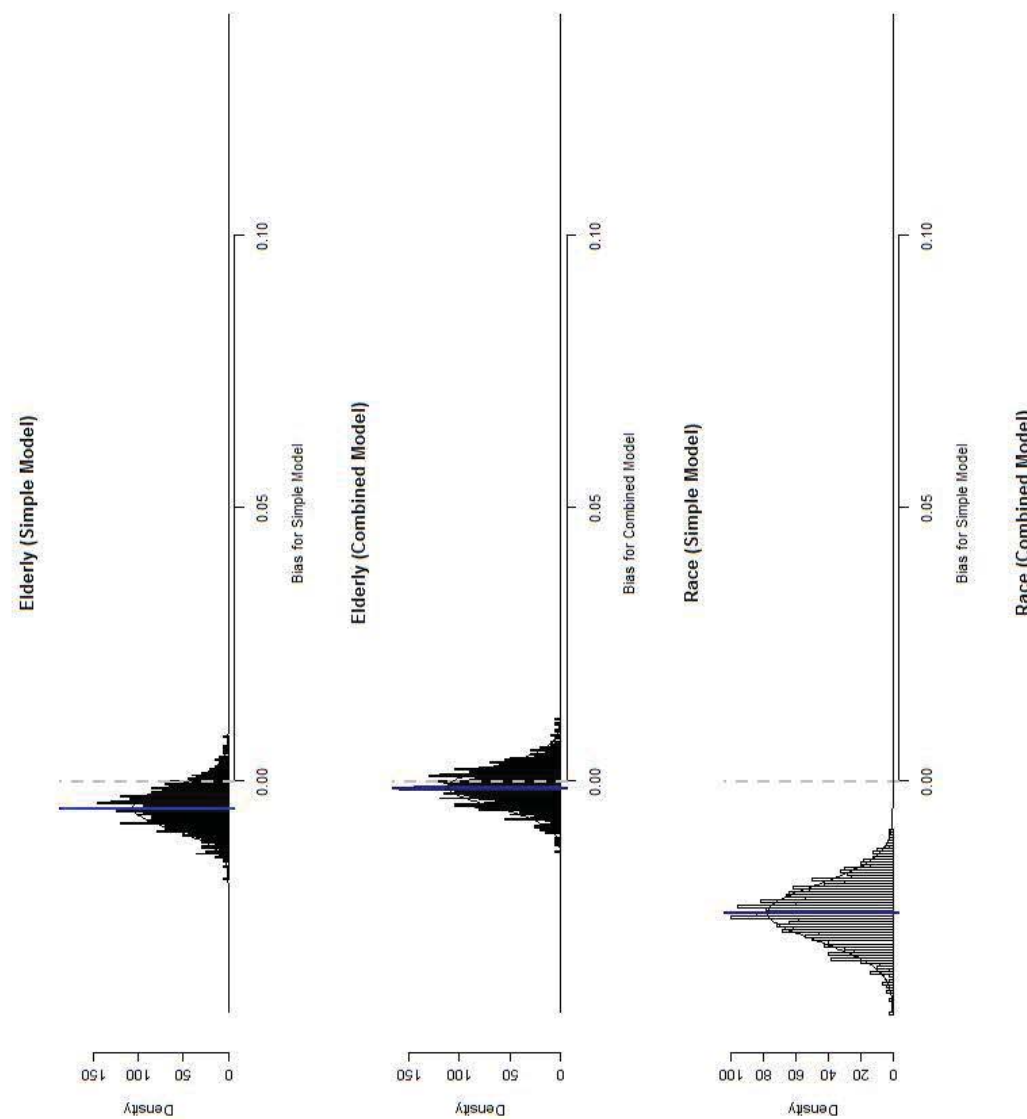

ป⿱艹
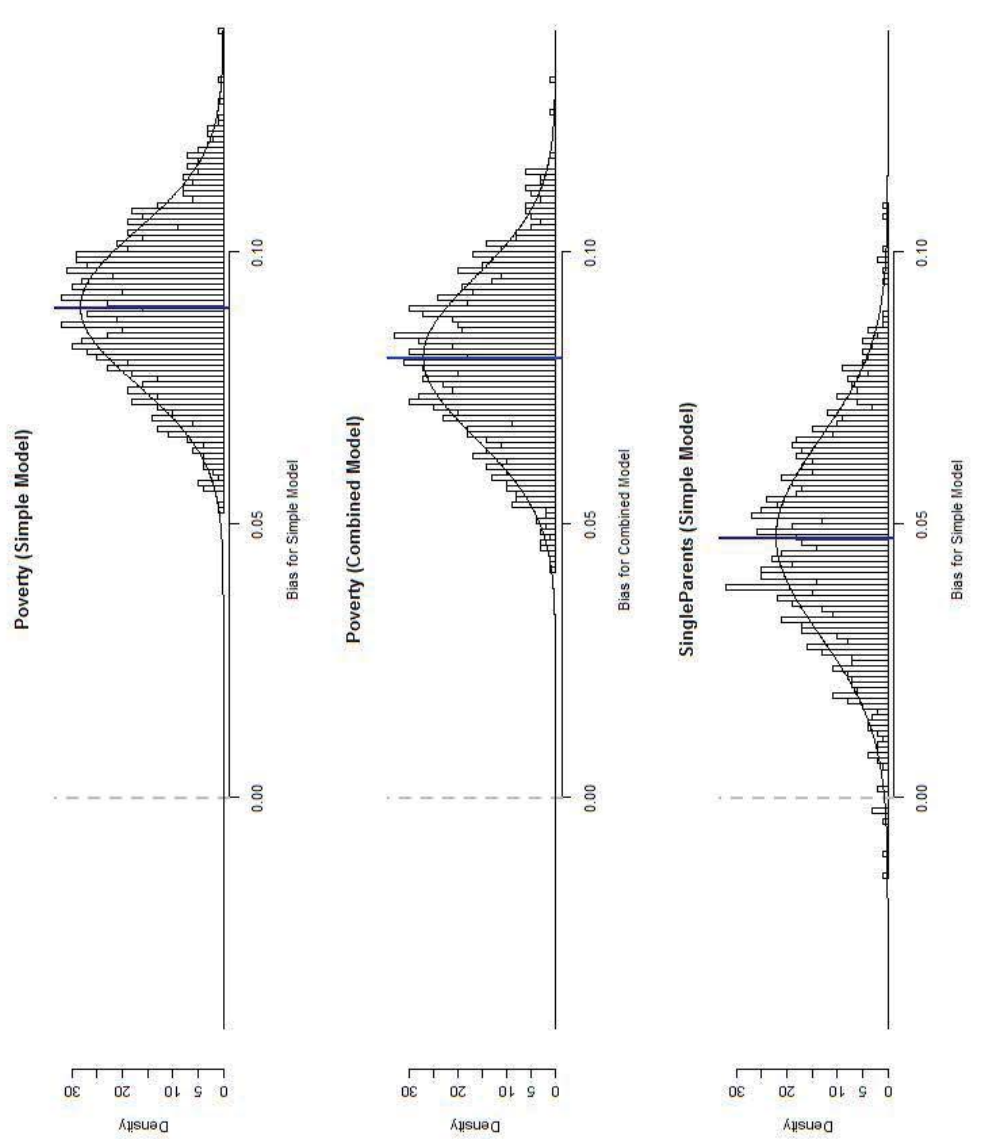

우 